\title{
TWELVE BRIDGES FROM A REDUCTIVE GROUP TO ITS LANGLANDS DUAL
}

\author{
G. LuszTiG
}

Introduction. These notes are based on a series of lectures given by the author at the Central China Normal University in Wuhan (July 2007). The aim of the lectures was to provide an introduction to the Langlands philosophy.

According to a theorem of Chevalley, connected reductive split algebraic groups over a fixed field $A$ are in bijection with certain combinatorial objects called root systems. Now there is a natural involution on the collection of root systems in which roots and coroots are interchanged. This corresponds by Chevalley's theorem to an involution on the collection of connected reductive split algebraic groups over $A$. The image of a group under this involution is called the Langlands dual of that group.

In the 1960's Langlands made the remarkable discovery that some features about the representations of a reductive group (such as classification) should be recorded in terms of data in the Langlands dual group. He thus formulated two conjectures: one involving groups over a local field and one involving automorphic representations with respect to a group over a global field.

In these notes we try to give several examples of "bridges" which connect some aspect of the collection $\left(G_{A}\right)$ of Chevalley groups attached to a root system $\mathcal{R}$ and to various commutative rings $A$ and some aspect of the analogous collection $\left(G_{A}^{*}\right)$ of Chevalley groups attached to the dual root system $\mathcal{R}^{*}$ and to various commutative rings $A$. By "aspect" we mean something about the structure of one of the groups $G_{A}$ or of its representations or of an associated object such as the affine Hecke algebra $\mathcal{H}$. In each case the existence of the bridge is surprising due to the fact that $\left(G_{A}\right)$ and $\left(G_{A}^{*}\right)$ are related only through a very weak connection (via their root systems); in particular there is no direct, elementary construction which produces the Langlands dual group from a given group.

In fact we describe twelve such bridges (some conjectural) the first three of which are very famous and were found by Langlands himself.

(I) A (conjectural) bridge from irreducible admissible representations of $G_{K}$ (where $K$ is a finite extension of $\mathbf{Q}_{p}$ ) to certain conjugacy classes of homomorphisms of the Weil group $W_{K}$ to $G_{\mathbf{C}}^{*}$. (See $\S 10$.) This bridge contains almost 
as a special case a bridge from irreducible representations of $\mathcal{H}$ specialized at a non-root of 1 and conjugacy classes of certain pairs of elements in $G_{\mathbf{C}}^{*}$. (See $\S 9$.)

(II) A bridge from irreducible admissible representations of $G_{\mathbf{R}}$ to certain conjugacy classes of homomorphisms of the Weil group $W_{\mathbf{R}}$ to $G_{\mathbf{C}}^{*}$. (See $\S 11$.)

(III) A (conjectural) bridge connecting certain automorphic representations attached to $G_{k}$ ( $k$ a function field over $\left.\mathbf{F}_{p}\right)$ and certain homomorphisms of $\operatorname{Gal}(\bar{k} / k)$ into $G_{\mathbf{C}}^{*} \cdot($ See $\S 12$.)

(IV) A bridge from unipotent classes in $G_{\mathbf{C}}^{*}$ to cells in the affine Weyl group constructed from $\mathcal{H}$. (See $\S 13$.)

(V) A bridge from "special unipotent pieces" in $G_{\overline{\mathbf{F}}_{p}}$ to "special unipotent pieces" in $G_{\overline{\mathbf{F}}_{p}}^{*} \cdot($ See $\S 14$.

(VI) A bridge from irreducible representations of $G_{\mathbf{F}_{q}}$ to certain "special" conjugacy classes in $G_{\mathbf{C}}^{*}$. (See $\left.\S 16.\right)$

(VII) A bridge from character sheaves on $G_{\mathbf{C}}$ to "special" conjugacy classes in $G_{\mathbf{C}}^{*} \cdot($ See $\oint 17$.

(VIII) A bridge constructed by Vogan connecting certain intersection cohomology spaces associated to symmetric spaces of $G_{\mathbf{C}}$ and similar objects for $G_{\mathbf{C}}^{*}$. (See $\S 18$.

(IX) A (partly conjectural) bridge connecting multiplicities in standard modules of $G_{K}$ (as in (II)) or $G_{\mathbf{R}}$ with intersection cohomology spaces arising from the geometry of $G_{\mathbf{C}}^{*}$. (See $\S 19$.)

(X) A bridge connecting the tensor product of two irreducible finite dimensional representations of $G_{\mathbf{C}}$ with the convolution of certain perverse sheaves on the affine Grassmannian attached to $G_{\mathbf{C}((\epsilon))}^{*}$. (See $\S 20$.)

(XI) A bridge connecting the canonical basis of the plus part of the enveloping algebra attached to $G_{\mathbf{Q}}$ with certain subsets of the totally positive part of the upper triangular subgroup of $G_{A}^{*}$ where $A=\mathbf{R}[[\epsilon]]$. (See $\S 21$.)

(XII) A (partly conjectural) bridge connecting the characters of irreducible modular representations of $G_{\overline{\mathbf{F}}_{p}}$ with certain intersection cohomology spaces associated with the geometry of $G_{\mathbf{C}((\epsilon))}^{*}$. (See $\S 22$.)

Note that in some cases (such as the very important Case III) our treatment is only a very brief sketch. Moreover to simplify the exposition we restrict ourselves to the case of split groups.

We now describe the contents of these notes. In $\S 1$ we introduce root systems. In $\S 2$ we use an idea of McKay (extended by Slodowy) to construct the irreducible simply connected root systems. In $\S 3$ we introduce the affine Weyl group. In $\S 4$ we introduce the affine Hecke algebra and its asymptotic version [L9]. In $\S 5$ we define the $\mathbf{Z}$-form of the coordinate ring of a Chevalley group. We do not follow the original approach of $[\mathrm{C}]$ but rather the approach of Kostant [Ko]. In $\S 6$ we define the Chevalley groups. In $\S 7$ we define the Weyl modules. In $\S 8$ we define the Langlands dual group. In $§ 9-\S 22$ we discuss the various bridges mentioned above.

1. Root systems. A root system is a collection 


$$
\mathcal{R}=\left(Y, X,\langle,\rangle, \check{\alpha}_{i}, \alpha_{i}(i \in I)\right)
$$

where $Y, X$ are finitely generated free abelian groups, $\langle\rangle:, Y \times X \rightarrow \mathbf{Z}$ is a perfect bilinear pairing, $I$ is a finite set, $\check{\alpha}_{i}(i \in I)$ are elements of $Y$ and $\alpha_{i}(i \in I)$ are elements of $X$ such that $\left\langle\check{\alpha}_{i}, \alpha_{i}\right\rangle=2$ for all $i,\left\langle\check{\alpha}_{i}, \alpha_{j}\right\rangle \in-\mathbf{N}$ for all $i \neq j$; it is assumed that the following (equivalent) conditions are satisfied:

(i) there exist $c_{i} \in \mathbf{Z}_{>0}(i \in I)$ such that the matrix $\left(c_{i}\left\langle\check{\alpha}_{i}, \alpha_{j}\right\rangle\right)_{i, j \in I}$ is symmetric, positive definite;

(ii) there exist $c_{i}^{\prime} \in \mathbf{Z}_{>0}(i \in I)$ such that the matrix $\left(\left\langle\check{\alpha}_{i}, \alpha_{j}\right\rangle c_{j}^{\prime}\right)_{i, j \in I}$ is symmetric, positive definite.

By the equivalence of (i),(ii),

$\mathcal{R}^{*}=\left(X, Y,\langle,\rangle^{\prime}, \alpha_{i}, \check{\alpha}_{i}(i \in I)\right)$ where $\langle x, y\rangle^{\prime}=\langle y, x\rangle$ for $x \in X, y \in Y$, is again a root system, said to be the dual of $\mathcal{R}$. Note that $\mathcal{R}^{* *}=\mathcal{R}$ in an obvious way.

For $\mathcal{R}$ as above let $W$ be the (finite) subgroup of $\operatorname{Aut}(Y)$ generated by the automorphisms $s_{i}: y \mapsto y-\left\langle y, \alpha_{i}\right\rangle \check{\alpha}_{i}(i \in I)$ or equivalently the subgroup of $\operatorname{Aut}(X)$ generated by the automorphisms $s_{i}: x \mapsto x-\left\langle\check{\alpha}_{i}, x\right\rangle \alpha_{i}(i \in I)$; these two subgroups may be identified by taking contragredients. We say that $W$ is the Weyl group of $\mathcal{R}$; it is also the Weyl group of $\mathcal{R}^{*}$. Let

$$
\begin{aligned}
& X^{+}=\left\{\lambda \in X ;\left\langle\check{\alpha}_{i}, \lambda\right\rangle \in \mathbf{N} \text { for all } i \in I\right\}, \\
& Y^{+}=\left\{y \in Y ;\left\langle y, \alpha_{i}\right\rangle \in \mathbf{N} \text { for all } i \in I\right\} .
\end{aligned}
$$

For $\lambda, \lambda^{\prime} \in X$ write $\lambda^{\prime} \geq \lambda$ if $\lambda^{\prime}-\lambda \in \sum_{i} \mathbf{N} \alpha_{i}$ and $\lambda^{\prime}>\lambda$ if $\lambda^{\prime} \geq \lambda, \lambda^{\prime} \neq \lambda$.

We say that $\mathcal{R}$ is simply connected if $Y=\sum_{i} \mathbf{Z} \check{\alpha}_{i}$. We say that $\mathcal{R}$ is adjoint if $X=\sum_{i} \mathbf{Z} \alpha_{i}$. We say that $\mathcal{R}$ is semisimple if $X / \sum_{i} \mathbf{Z} \alpha_{i}$ is finite or equivalently $Y / \sum_{i} \mathbf{Z} \check{\alpha}_{i}$ is finite. We say that $\mathcal{R}$ is irreducible if $I \neq \emptyset$ and there is no partition $I=I^{\prime} \cup I^{\prime \prime}$ of $I$ such that $I^{\prime}, I^{\prime \prime}$ are $\neq \emptyset$ and $\left\langle\check{\alpha}_{i}, \alpha_{j}\right\rangle=0$ for all $i \in I^{\prime}, j \in I^{\prime \prime}$.

2. Subgroups of $S L_{2}(\mathbf{C})$ and root systems. In [MK] McKay discovered a remarkable direct connection between finite subgroups of $S L_{2}(\mathbf{C})$ and "simply laced affine Dynkin diagrams". Slodowy [SL] extended this to a connection between certain pairs of subgroups of $S L_{2}(\mathbf{C})$ (one contained in the other) and "affine Dynkin diagrams".

Let $\Gamma, \Gamma^{\prime}$ be two finite subgroups of $S L_{2}(\mathbf{C})$ such that $\Gamma$ is a normal subgroup of $\Gamma^{\prime}$ with $\Gamma^{\prime} / \Gamma$ cyclic. We show how to attach to $\left(\Gamma, \Gamma^{\prime}\right)$ a root system (we use an argument generalizing one in $[\mathrm{L} 12,1.2])$. Let $\mathcal{X}$ be the category of finite dimensional complex representations of $\Gamma$ which can be extended to representations of $\Gamma^{\prime}$. Let $\rho_{i}(i \in \tilde{I})$ be the indecomposable objects of $\mathcal{X}$ up to isomorphism that is, the representations of $\Gamma$ which are restrictions of irreducible representations of $\Gamma^{\prime}$. Let $i_{0} \in \tilde{I}$ be such that $\rho_{i_{0}}$ is the trivial representation of $\Gamma$ on $\mathbf{C}$. Let $\sigma$ be the obvious representation of $\Gamma$ on $\mathbf{C}^{2}$; we have $\sigma \in \mathcal{X}$. Let $V$ be the $\mathbf{R}$-vector space with basis $\{i ; i \in \tilde{I}\}$. Any object $\rho$ of $\mathcal{X}$ gives rise to a vector $\rho=\sum_{i} n_{i} i \in V$ where $\rho \cong \oplus_{i} \rho_{i}^{\oplus n_{i}}$; here $n_{i} \in \mathbf{N}$. For $i \in \tilde{I}$ we have $\rho_{i} \otimes \sigma \in \mathcal{X}$ and $\underline{\rho_{i} \otimes \sigma}=\sum_{j \in \tilde{I}} c_{i j} j$ with $c_{i j} \in \mathbf{N}$.

For $i \in I, \rho_{i}$ is the direct sum of $m_{i}$ irreducible representations of $\Gamma$ (each with multiplicity 1). Let [,] be the bilinear form on $V$ with values in $\mathbf{R}$ given by 
$[i, j]=\left(2 \delta_{i j}-c_{i j}\right) m_{j}$ for $i, j \in \tilde{I}$. Let $x=\sum_{i} x_{i} i \in V, x^{\prime}=\sum_{i} x_{i}^{\prime} i \in V$ where $x_{i}, x_{i}^{\prime} \in \mathbf{R}$. For $\gamma \in \Gamma$ let $\lambda_{g}$ be an eigenvalue of $\gamma$ on $\mathbf{C}^{2}$. We have

$$
\begin{aligned}
& {\left[x, x^{\prime}\right]=|\Gamma|^{-1} \sum_{i, j ; \gamma \in \Gamma} x_{i} x_{j}^{\prime} \operatorname{tr}\left(\gamma, \rho_{i}\right) \overline{\operatorname{tr}\left(\gamma, \rho_{j}\right)}\left(2-\lambda_{g}-\overline{\lambda_{g}}\right)} \\
& =|\Gamma|^{-1} \sum_{\gamma \in \Gamma}\left(\sum_{i} x_{i} \operatorname{tr}\left(\gamma, \rho_{i}\right)\left|1-\lambda_{\gamma}\right|\right)\left(\sum_{j} x_{j}^{\prime} \overline{\operatorname{tr}\left(\gamma, \rho_{j}\right)}\left|1-\lambda_{\gamma}\right|\right) .
\end{aligned}
$$

In particular,

$$
[x, x]=|\Gamma|^{-1} \sum_{\gamma \in \Gamma}\left|\sum_{i} x_{i} \operatorname{tr}\left(\gamma, \rho_{i}\right)\right| 1-\left.\lambda_{\gamma}\right|^{2} \geq 0
$$

If $[x, x]=0$ then for any $\gamma \in \Gamma$ we have $\sum_{i} x_{i} \operatorname{tr}\left(\gamma, \rho_{i}\right)\left|1-\lambda_{\gamma}\right|=0$ that is, for any $\gamma \in \Gamma-\{1\}$ we have $\sum_{i} x_{i} \operatorname{tr}\left(\gamma, \rho_{i}\right)=0$ that is, there exists $c \in \mathbf{R}$ such that $x=c \underline{r}$ where $\mathfrak{r} \in \mathcal{X}$ is the regular representation of $\Gamma$; if in addition we have $x_{i_{0}}=0$ then we see that $c=0$ hence $x=0$.

Let $I=\tilde{I}-\left\{i_{0}\right\}$. Let $Y$ be the subgroup of $V$ generated by $\{i ; i \in I\}$. For $i \in I$ let $\check{\alpha}_{i}=i \in Y$. Let $X=\operatorname{Hom}(Y, \mathbf{Z})$. Let $\langle\rangle:, Y \times X \rightarrow \mathbf{Z}$ be the obvious pairing. For $j \in I$ define $\alpha_{j} \in X$ by $\left\langle\check{\alpha}_{i}, \alpha_{j}\right\rangle=(i, j)$. We have $\left\langle\check{\alpha}_{i}, \alpha_{i}\right\rangle=$ 2 for all $i \in I$ and $\left\langle\check{\alpha}_{i}, \alpha_{j}\right\rangle=-c_{i j} \in-\mathbf{N}$ for $i \neq j$ in $I$. By the argument above the matrix $\left(\left\langle\check{\alpha}_{i}, \alpha_{j}\right\rangle m_{j}\right)_{i, j \in I}$ is symmetric and positive definite. Hence $\mathcal{R}=$ $\left(Y, X,\langle\rangle,, \check{\alpha}_{i}, \alpha_{i}(i \in I)\right)$ is a (simply connected) root system.

Note that all simply connected irreducible root systems are obtained by this construction exactly once (up to isomorphism) from pairs $\left(\Gamma, \Gamma^{\prime}\right)$ as above (up to conjugacy) with $\Gamma \neq\{1\}$ and with the property that any element of $\Gamma^{\prime}$ which commutes with any element of $\Gamma$ is contained in $\Gamma$. Such pairs are classified as follows:

(a) $\Gamma=\Gamma^{\prime}$ is a cyclic group $\mathbf{Z}_{n}$ of order $n \geq 2$;

(b) $\Gamma=\Gamma^{\prime}$ is a binary dihedral group $\mathcal{D}_{4 n}$ of order $4 n \geq 8$;

(c) $\Gamma=\Gamma^{\prime}$ is a binary tetrahedral group $G_{24}$ of order 24;

(d) $\Gamma=\Gamma^{\prime}$ is a binary octahedral group $G_{48}$ of order 48 ;

(e) $\Gamma=\Gamma^{\prime}$ is a binary icosahedral group $G_{120}$ of order 120 ;

(f) $\Gamma^{\prime}=\mathcal{D}_{4 n}, \Gamma=\mathbf{Z}_{2 n}$ with $n \geq 2$;

(g) $\Gamma^{\prime}=\mathcal{D}_{8 n}, \Gamma=\mathcal{D}_{4 n}$ with $n \geq 2$;

(h) $\Gamma^{\prime}=G_{48}, \Gamma=G_{24}$;

(i) $\Gamma^{\prime}=G_{24}, \Gamma=\mathcal{D}_{8}$.

3. Affine Weyl group. Let $\mathcal{R}=\left(Y, X,\langle\rangle,, \check{\alpha}_{i}, \alpha_{i}(i \in I)\right)$ be a root system. Let $W, s_{i}(i \in I)$ be as in $\S 1$. Let $\tilde{W}$ be the semidirect product $W \cdot Y$. We have $\tilde{W}=\left\{w a^{y} ; w \in W, y \in Y\right\}$ where $a$ is a symbol; the multiplication is given by $\left(w a^{y}\right)\left(w^{\prime} a^{y^{\prime}}\right)=w w^{\prime} a^{w^{-1}(y)+y^{\prime}}$ for $w, w^{\prime} \in W, y, y^{\prime} \in Y$. We identify $Y$ with its image under the homomorphism $y \mapsto 1 a^{y}$ (a normal subgroup of $\tilde{W}$ ) and $W$ with its image under the homomorphism $w \mapsto w a^{0}$. 
Let $R$ be the set of elements of $X$ of the form $w\left(\alpha_{i}\right)$ for some $w \in W, i \in I$. Let $\check{R}$ be the set of elements of $Y$ of the form $w\left(\check{\alpha}_{i}\right)$ for some $w \in W, i \in I$. There is a unique $W$-equivariant bijection $\alpha \leftrightarrow \check{\alpha}$ between $R$ and $\check{R}$ such that $\alpha_{i} \leftrightarrow \check{\alpha}_{i}$ for any $i \in I$. For $\alpha \in R$ we set $s_{\alpha}=w s_{i} w^{-1} \in W$ where $\alpha=w\left(\alpha_{i}\right), w \in W, i \in I$. Note that $s_{\alpha}$ is well defined. Let $R_{\min }$ be the set of all $\alpha \in R$ such that the following holds: if $\alpha^{\prime} \in R, \alpha^{\prime} \leq \alpha$ then $\alpha^{\prime}=\alpha$.

Let $R^{+}=R \cap\left(\sum_{i} \mathbf{N} \alpha_{i}\right), R^{-}=-R^{+}$. We have $R=R^{+} \cup R^{-}$. Following Iwahori and Matsumoto [IM], we define a function $l: \tilde{W} \rightarrow \mathbf{N}$ by

$$
l\left(w a^{y}\right)=\sum_{\alpha \in R^{+} ; w(\alpha) \in R^{-}}|\langle y, \alpha\rangle+1|+\sum_{\alpha \in R^{+} ; w(\alpha) \in R^{+}}|\langle y, \alpha\rangle| .
$$

Let $S$ be the subset of $W$ consisting of the involutions $s_{i}(i \in I)$ and the involutions $s_{\alpha} a^{\check{\alpha}}$ with $\alpha \in R_{\text {min }}$. Note that $\left.l\right|_{S}=1$.

If $y \in Y^{+}$we have $l\left(a^{y}\right)=\sum_{\alpha \in R^{+}}\langle y, \alpha\rangle$. Hence for $y, y^{\prime} \in Y^{+}$we have $l\left(a^{y} \cdot a^{y^{\prime}}\right)=l\left(a^{y+y^{\prime}}\right)=l\left(a^{y}\right)+l\left(a^{y^{\prime}}\right)$.

4. Affine Hecke algebra. We preserve the notation of $\S 3$. Let $\mathcal{A}=\mathbf{Z}\left[v, v^{-1}\right]$ where $v$ is an indeterminate. Let $\mathcal{H}$ be the associative $\mathcal{A}$-algebra with 1 with generators $T_{w}(w \in \tilde{W})$ and relations

$\left(T_{s}-v\right)\left(T_{s}+v^{-1}\right)=0$ for $s \in S$,

$T_{w} T_{w^{\prime}}=T_{w w^{\prime}}$ for $w, w^{\prime} \in \tilde{W}$ such that $l(w)+l\left(w^{\prime}\right)=l\left(w w^{\prime}\right)$.

We have $T_{1}=1$ and $\left\{T_{w} ; w \in \tilde{W}\right\}$ is an $\mathcal{A}$-basis of $\mathcal{H}$. Let $h \mapsto h^{\dagger}$ be the $\mathcal{A}$ algebra involution of $\mathcal{H}$ such that $T_{w}^{\dagger}=(-1)^{l(w)} T_{w}^{-1}$. Let $h \mapsto \bar{h}$ be the ring involution of $\mathcal{H}$ such that $\overline{T_{w}}=T_{w^{-1}}^{-1}$ for $w \in \tilde{W}$ and $\overline{v^{n}}=v^{-n}$ for $n \in \mathbf{Z}$. Let $z \in \tilde{W}$. According to [KL1] there is a unique element $c_{z} \in \mathcal{H}$ such that $\overline{c_{z}}=c_{z}$ and $c_{z}=\sum_{w \in \tilde{W}} p_{w, z} T_{w}$ where $p_{w, z} \in v^{-1} \mathbf{Z}\left[v^{-1}\right]$ for all $w \neq z, p_{z, z}=1$ and $p_{w, z}=0$ for all but finitely many $w$. Note that $\left\{c_{w} ; w \in \tilde{W}\right\}$ is an $\mathcal{A}$-basis of $\mathcal{H}$. For $x, y \in \tilde{W}$ we write $c_{x} c_{y}=\sum_{z \in \tilde{W}} h_{x, y, z} c_{z}$ where $h_{x, y, z} \in \mathcal{A}$ is 0 for all but finitely many $z$. Let $z \in \tilde{W}$. According to [L9] there is a unique $a(z) \in \mathbf{N}$ such that $h_{x, y, z} \in v^{a(z)} \mathbf{Z}\left[v^{-1}\right]$ for any $x, y$ and $h_{x, y, z} \notin v^{a(z)-1} \mathbf{Z}\left[v^{-1}\right]$ for some $x, y$. We have $h_{x, y, z}=\gamma_{x, y, z^{-1}} v^{a(z)} \bmod v^{a(z)-1} \mathbf{Z}\left[v^{-1}\right]$ where $\gamma_{x, y, z^{-1}} \in \mathbf{Z}$. Let $J$ be the free abelian group with basis $\left\{t_{w} ; w \in \mathbf{W}\right\}$. Consider the $\mathbf{Z}$-algebra structure on $J$ such that $t_{x} t_{y}=\sum_{z \in \tilde{W}} \gamma_{x, y, z^{-1}} t_{z}$ for all $x, y$ in $\tilde{W}$. This structure is associative and the ring $J$ has a unit element 1 of the form $\sum_{d \in \mathcal{D}} t_{d}$ where $\mathcal{D}$ is a finite subset of $\tilde{W}$ consisting of involutions [L9]. For $x, y$ in $\tilde{W}$ we write $x \sim y$ when $t_{x} t_{u} t_{y} \neq 0$ for some $u \in \tilde{W}$. This is an equivalence relation on $\tilde{W}$; the equivalence classes are called two-sided cells. For any two-sided cell $c$ let $J_{c}$ be the subgroup of $J$ spanned by $\left\{t_{z} ; z \in c\right\}$. This is a subring of $J$ with unit $\sum_{d \in \mathcal{D} \cap c} t_{d}$. We have $J=\oplus_{c} J_{c}$ as rings. Consider the $\mathcal{A}$-linear map $\phi: \mathcal{H} \rightarrow \mathcal{A} \otimes J$ given by

$$
\phi\left(c_{x}^{\dagger}\right)=\sum_{z \in \tilde{W}, d \in \mathcal{D} ; a(d)=a(z)} h_{x, d, z} t_{z}
$$

for any $x \in \tilde{W}$. This is an $\mathcal{A}$-algebra homomorphism [L9]. 
5. Coordinate ring. Let $\mathcal{R}=\left(Y, X,\langle\rangle,, \check{\alpha}_{i}, \alpha_{i}(i \in I)\right)$ be a root system. Let $f$ be the associative $\mathbf{Q}$-algebra with 1 defined by the generators $\theta_{i}(i \in I)$ and the Serre relations

$$
\sum_{a, b \in \mathbf{N} ; a+b=1-\left\langle\check{\alpha}_{i}, \alpha_{j}\right\rangle}(-1)^{a}\left(\theta_{i}^{a} / a !\right) \theta_{j}\left(\theta_{i}^{b} / b !\right)
$$

for $i \neq j$ in $I$. Let ${ }^{0} \underline{U}$ be the symmetric algebra of $\mathbf{Q} \otimes Y$. Let $\underline{U}$ be the $\mathbf{Q}$-algebra with 1 defined by the generators $x^{+}, x^{-}(x \in \underline{f}), \underline{a} \in{ }^{0} \underline{U}$ and the relations:

$x \mapsto x^{+}$is an algebra homomorphism $\underline{f} \rightarrow \underline{U}$ respecting 1 ;

$x \mapsto x^{-}$is an algebra homomorphism $\bar{f} \rightarrow \underline{U}$ respecting 1 ;

$\underline{a} \mapsto \underline{a}$ is an algebra homomorphism ${ }^{0} \underline{U} \rightarrow \underline{U}$ respecting 1 ;

$y \theta_{i}^{+}-\theta_{i}^{+} y=\left\langle y, \alpha_{i}\right\rangle \theta_{i}^{+}$for $y \in Y, i \in \bar{I}$

$y \theta_{i}^{-}-\theta_{i}^{-} y=-\left\langle y, \alpha_{i}\right\rangle \theta_{i}^{-}$for $y \in Y, i \in I$

$\theta_{i}^{+} \theta_{j}^{-}-\theta_{j}^{-} \theta_{i}^{+}=\delta_{i j} \check{\alpha}_{i}$ for $i, j$ in $I$.

Define a Q-algebra homomorphism $\Delta: \underline{U} \rightarrow \underline{U} \otimes \underline{U}$ by $\Delta\left(\theta_{i}^{+}\right)=\theta_{i}^{+} \otimes 1+1 \otimes \theta_{i}^{+}$, $\Delta\left(\theta_{i}^{-}\right)=\theta_{i}^{-} \otimes 1+1 \otimes \theta_{i}^{-}$for $i \in I, \Delta(y)=y \otimes 1+1 \otimes y$ for $y \in Y$. Define a Q-algebra isomorphism $\Sigma: \underline{U} \rightarrow \underline{U}^{\text {opp }}$ by $\Sigma\left(\theta_{i}^{+}\right)=-\theta_{i}^{+}, \Sigma\left(\theta_{i}^{-}\right)=-\theta_{i}^{-}$for $i \in I$, $\Sigma(y)=-y$ for $y \in Y$.

Let $\underline{f}_{\mathbf{Z}}$ be the subring of $\underline{f}$ generated by the elements $\theta_{i}^{(n)}:=\theta_{i}^{n} / n !, \quad(i \in$ $I, n \in \overline{\mathbf{N}})$. Note that $\underline{f}_{\mathbf{Z}}$ is a lattice in the $\mathbf{Q}$-vector space $\underline{f}$. Following [Ko] we define $\underline{U}_{\mathbf{Z}}$ to be the subring of $\underline{U}$ generated by the elements $x^{+}, x^{-}\left(x \in \underline{f}_{\mathbf{Z}}\right)$ and $\left(\begin{array}{l}y \\ n\end{array}\right):=\frac{y(y-1) \ldots(y-n+1)}{n !},(y \in Y, n \in \mathbf{N})$. Note that $\underline{U}_{\mathbf{Z}}$ is a lattice in the $\mathbf{Q}$-vector space $\underline{U}$. Hence $\underline{U}_{\mathbf{Z}} \otimes_{\mathbf{Z}} \underline{U}_{\mathbf{Z}}$ is a lattice in $\underline{U} \otimes_{\mathbf{Q}} \underline{U}$. Note that $\Delta$ restricts to a ring homomorphism $\underline{U}_{\mathbf{Z}} \rightarrow \underline{U}_{\mathbf{Z}} \otimes_{\mathbf{Z}} \underline{U}_{\mathbf{Z}}$ denoted again by $\Delta$. Also $\Sigma$ restricts to a ring isomorphism $\underline{U}_{\mathbf{Z}} \rightarrow \underline{U}_{\mathbf{Z}}^{\text {opp }}$ denoted again by $\Sigma$.

For any $\underline{U}$-module $M$ and any $x \in X$ we set

$M^{x}=\{m \in M ; y m=\langle y, x\rangle m$ for any $y \in Y\}$.

Let $\mathcal{C}$ be the category whose objects are $\underline{U}$-modules $M$ with $\operatorname{dim}_{\mathbf{Q}} M<\infty$ such that $M=\oplus_{x \in X} M^{x}$. For any $\mathbf{Q}$-vector space $V$ we set $V^{\dagger}=\operatorname{Hom}_{\mathbf{Q}}(V, \mathbf{Q})$. For $M \in \mathcal{C}$ we define $c_{M}: M \otimes M^{\dagger} \rightarrow \underline{U}^{\dagger}$ by $m \otimes \xi \mapsto[u \mapsto \xi(u m)]$. Let

$$
\mathcal{O}=\sum_{M \in \mathcal{C}} c_{M}\left(M \otimes M^{\dagger}\right)
$$

a Q-subspace of $\underline{U}^{\dagger}$. (This agrees with the definition in [Ko] when $\mathcal{R}$ is semisimple.) For $f, f^{\prime} \in \mathcal{O}$ we define $f f^{\prime}: \underline{U} \rightarrow \mathbf{Q}$ by $u \mapsto \sum_{s} f\left(u_{s}\right) f^{\prime}\left(u_{s}^{\prime}\right)$ where $\Delta(u)=\sum_{s} u_{s} \otimes u_{s}^{\prime}, u_{s}, u_{s}^{\prime} \in \underline{U}$. We have $f f^{\prime} \in \mathcal{O}$. This defines a structure of associative, commutative algebra on $\mathcal{O}$. This algebra has a unit element: the algebra homomorphism $\underline{U} \rightarrow \mathbf{Q}$ such that $\theta_{i}^{+} \mapsto 0, \theta_{i}^{-} \mapsto 0$ for $i \in I, y \mapsto 0$ for $y \in Y$ and $1 \mapsto 1$. For $f \in \mathcal{O}$ we define a linear function $\delta(f): \underline{U} \otimes \underline{U} \rightarrow \mathbf{Q}$ by $u_{1} \otimes u_{2} \mapsto f\left(u_{1} u_{2}\right)$. Note that $\mathcal{O} \otimes \mathcal{O}$ is naturally a subspace of $(\underline{U} \otimes \underline{U})^{\dagger}$ and that the image of $\delta: \mathcal{O} \rightarrow(\underline{U} \otimes \underline{U})^{\dagger}$ is contained in the subspace $\mathcal{O} \otimes \mathcal{O}$ so that $\delta$ defines a linear map $\mathcal{O} \rightarrow \mathcal{O} \otimes \mathcal{O}$ denoted again by $\delta$. This is an algebra homomorphism. 
For $f \in \mathcal{O}$ we define a linear function $\sigma(f): \underline{U} \rightarrow \mathbf{Q}$ by $u \mapsto f(\Sigma(u))$. We have $\sigma(f) \in \mathcal{O}$; thus $\sigma$ defines a linear map $\mathcal{O} \rightarrow \mathcal{O}$ denoted again by $\sigma$. This is an algebra homomorphism. Define $\epsilon: \mathcal{O} \rightarrow \mathbf{Q}$ by $f \mapsto f(1)$. Note that the commutative algebra $\mathcal{O}$ with the comultiplication $\delta$, the antipode $\sigma$ and the counit $\epsilon$ is a Hopf algebra over $\mathbf{Q}$.

Let $f \in \mathcal{O}$. There is a unique collection $\left(f^{x}\right)_{x \in X}$ of numbers in $\mathbf{Q}$ such that $f^{x}=0$ for all but finitely many $x \in X$ and

$$
f\left(y_{1}^{n_{1}} y_{2}^{n_{2}} \ldots y_{r}^{n_{r}}\right)=\sum_{x \in X}\left\langle y_{1}, x\right\rangle^{n_{1}}\left\langle y_{2}, x\right\rangle^{n_{2}} \ldots\left\langle y_{r}, x\right\rangle^{n_{r}} f^{x}
$$

for any $y_{1}, y_{2}, \ldots, y_{r}$ in $Y$ and $n_{1}, n_{2}, \ldots, n_{r}$ in $\mathbf{N}$. For example if $f=c_{M}(m \otimes \xi)$ where $M \in \mathcal{C}, m \in M, \xi \in M^{\dagger}$, we have $f^{x}=\xi\left(m_{x}\right)$ where $m_{x} \in M^{x}$ are defined by $m=\sum_{x \in X} m_{x}$. Note that for any $x \in X, f \mapsto f^{x}$ is a linear function $\mathcal{O} \rightarrow \mathbf{Q}$.

Let $\mathcal{O}_{\mathbf{Z}}=\left\{f \in \mathcal{O} ; f\left(\underline{U}_{\mathbf{Z}}\right) \subset \mathbf{Z}\right\}$. (This agrees with the definition in [Ko] when $\mathcal{R}$ is semisimple.) Note that $\mathcal{O}_{\mathbf{Z}}$ is a subring of $\mathcal{O}$. One can show that $\mathcal{O}_{\mathbf{z}}$ is a lattice in the $\mathbf{Q}$-vector space $\mathcal{O}$. Hence $\mathcal{O}_{\mathbf{Z}} \otimes_{\mathbf{Z}} \mathcal{O}_{\mathbf{Z}}$ is a lattice in the $\mathbf{Q}$-vector space $\mathcal{O} \otimes \mathcal{O}$. Note that $\delta: \mathcal{O} \rightarrow \mathcal{O} \otimes \mathcal{O}$ restricts to a ring homomorphism $\delta_{\mathbf{Z}}: \mathcal{O}_{\mathbf{Z}} \rightarrow \mathcal{O}_{\mathbf{Z}} \otimes_{\mathbf{Z}} \mathcal{O}_{\mathbf{Z}}$ $\sigma: \mathcal{O} \rightarrow \mathcal{O}$ restricts to a ring isomorphism $\sigma_{\mathbf{Z}}: \mathcal{O}_{\mathbf{Z}} \rightarrow \mathcal{O}_{\mathbf{Z}} ; \epsilon: \mathcal{O} \rightarrow \mathbf{Q}$ restricts to a ring homomorphism $\epsilon_{\mathbf{Z}}: \mathcal{O}_{\mathbf{Z}} \rightarrow \mathbf{Z}$. The commutative ring $\mathcal{O}_{\mathbf{Z}}$ together with the comultiplication $\delta_{\mathbf{Z}}$, the antipode $\sigma_{\mathbf{Z}}$ and the counit $\epsilon_{\mathbf{Z}}$ is a Hopf ring. For any $x \in X$ and $f \in \mathcal{O}_{\mathbf{Z}}$ we have $f^{x} \in \mathbf{Z}$.

For any commutative ring $A$ with 1 we set $\mathcal{O}_{A}=A \otimes \mathcal{O}_{\mathbf{Z}}$. By extension of scalars, from $\delta_{\mathbf{Z}}, \sigma_{\mathbf{Z}}, \epsilon_{\mathbf{Z}}$ we get $A$-algebra homomorphisms $\delta_{A}: \mathcal{O}_{A} \rightarrow \mathcal{O}_{A} \otimes_{A} \mathcal{O}_{A}$, $\sigma_{A}: \mathcal{O}_{A} \rightarrow \mathcal{O}_{A}, \epsilon_{A}: \mathcal{O}_{A} \rightarrow A$. The commutative $A$-algebra $\mathcal{O}_{A}$ together with the comultiplication $\delta_{A}$, the antipode $\sigma_{A}$ and the counit $\epsilon_{A}$ is a Hopf algebra over $A$. For any $x \in X$ the homomorphism $\mathcal{O}_{\mathbf{Z}} \rightarrow \mathbf{Z}$ gives rise by extension of scalars to an $A$-linear map $\mathcal{O}_{A} \rightarrow A$ denoted again by $f^{x}$.

The following two properties are proved in [L18]:

(i) the $A$-algebra $\mathcal{O}_{A}$ is finitely generated;

(ii) if $A$ is an integral domain then $\mathcal{O}_{A}$ is an integral domain.

6. Chevalley groups. We preserve the notation of $\S 5$. Let $W, s_{i}$ be as in $\S 1$. Let $A$ be a commutative ring with 1 . As in [Ko] we define $G_{A}$ to be the set of $A$-algebra homomorphisms $\mathcal{O}_{A} \rightarrow A$ respecting 1 . For $g, g^{\prime} \in G_{A}$ we define $g g^{\prime}: \mathcal{O}_{A} \rightarrow A$ by $f \mapsto \sum_{s} g\left(f_{s}\right) g^{\prime}\left(f_{s}^{\prime}\right)$ where $\delta_{A}(f)=\sum_{s} f_{s} \otimes f_{s}^{\prime}$ with $f_{s}, f_{s}^{\prime}$ in $\mathcal{O}_{A}$. Then $g g^{\prime} \in G_{A}$ and $\left(g, g^{\prime}\right) \mapsto g g^{\prime}$ is a group structure on $G_{A}$ with unit element $\epsilon_{A}$. We say that $G_{A}$ is the Chevalley group attached to the root system $\mathcal{R}$ and to the commutative ring $A$. We write also $G_{A}^{\mathcal{R}}$ instead of $G_{A}$ when we want to emphasize the dependence on $\mathcal{R}$.

If $\kappa: A \rightarrow A^{\prime}$ is a homomorphism of commutative rings with 1 and $g: \mathcal{O}_{A} \rightarrow A$ is in $G_{A}$ then applying to $g$ the functor $A^{\prime} \otimes_{A}$ ? (where $A^{\prime}$ is regarded as an $A$ algebra via $\kappa$ ) we obtain an $A^{\prime}$-algebra homomorphism $\mathcal{O}_{A^{\prime}} \rightarrow A^{\prime}$ respecting 1 which is denoted by $\tilde{g}$. Now $g \mapsto \tilde{g}$ is a group homomorphism $G_{A} \rightarrow G_{A^{\prime}}$ said to be induced by $\kappa$. 
For any $i \in I, b \in A$ we define an $A$-linear map $x_{i}(b): \mathcal{O}_{A} \rightarrow A$ by

$$
\sum_{s} a_{s} \otimes f_{s} \mapsto \sum_{s} \sum_{n \in \mathbf{N}} a_{s} b^{n} f_{s}\left(\left(\theta_{i}^{(n)}\right)^{+}\right)
$$

Here $a_{s} \in A, f_{s} \in \mathcal{O}_{\mathbf{z}}$. Since $f_{s} \in \mathcal{O}$ we have $f_{s}\left(\left(\theta_{i}^{(n)}\right)^{+}\right)=0$ for large enough $n$ so that the last sum is finite. From the definitions we see that $x_{i}(b) \in G_{A}$ and that $b \mapsto x_{i}(b)$ is an (injective) group homomorphism $A \rightarrow G_{A}$.

Similarly, for any $i \in I, b \in A$ we define an $A$-linear map $y_{i}(b): \mathcal{O}_{A} \rightarrow A$ by

$$
\sum_{s} a_{s} \otimes f_{s} \mapsto \sum_{s} \sum_{n \in \mathbf{N}} a_{s} b^{n} f_{s}\left(\left(\theta_{i}^{(n)}\right)^{-}\right)
$$

Here $a_{s} \in A, f_{s} \in \mathcal{O}_{\mathbf{z}}$. Again the last sum is finite. From the definitions we see that $y_{i}(b) \in G_{A}$ and that $b \mapsto y_{i}(b)$ is an (injective) group homomorphism $A \rightarrow G_{A}$.

Let $A^{*}$ be the group of units of $A$. Let $t=\sum_{r} a_{r} \otimes y_{r} \in A^{*} \otimes Y$ with $a_{r} \in$ $A^{*}, y_{r} \in Y$. We define $\underline{t}: \mathcal{O}_{A} \rightarrow A$ by

$$
f \mapsto \sum_{r} \sum_{x \in X} a_{r}^{\left\langle y_{r}, x\right\rangle} f^{x}
$$

(Since $a_{r} \in A^{*}, a_{r}^{n}$ is defined for any $n \in \mathbf{Z}$.) From the definitions we see that $\underline{t} \in G_{A}$ and that $t \mapsto \underline{t}$ is an injective group homomorphism $A^{*} \otimes Y \rightarrow G_{A}$ with image denoted by $T_{A}$ (a commutative subgroup of $G_{A}$ ). We identify $A^{*} \otimes Y$ with $T_{A}$ via this homomorphism. We write also $T_{A}^{\mathcal{R}}$ instead of $T_{A}$ when we want to emphasize the dependence on $\mathcal{R}$.

For $i \in I$ we define an element $\dot{s}_{i} \in G_{A}$ by $\dot{s}_{i}=x_{i}(1) y_{i}(-1) x_{i}(1)$. We have $\dot{s}_{i}^{2}=(-1) \otimes \check{\alpha}_{i} \in A^{*} \otimes Y=T_{A}$. Moreover, for $i \neq j$ we have $\dot{s}_{i} \dot{s}_{j} \dot{s}_{i} \cdots=\dot{s}_{j} \dot{s}_{i} \dot{s}_{j} \ldots$ (both sides have $n$ factors where $n$ is the order of $s_{i} s_{j}$ in $W$ ). It follows that for any $w \in W$ there is a well defined element $\dot{w} \in G_{A}$ such that $\dot{w}=\dot{s}_{i_{1}} \dot{s}_{i_{2}} \ldots \dot{s}_{i_{r}}$ whenever $w=s_{i_{1}} s_{i_{2}} \ldots s_{i_{r}}$ with $r=l(w)$. Note that $\dot{w} T_{A} \dot{w}^{-1}=T_{A}$. More precisely, for $t \in T_{A}$ we have $\dot{w} t \dot{w}^{-1}=w(t)$ where $w: t \mapsto w(t)$ is the $W$-action on $T_{A}$ given by $a \otimes y \mapsto a \otimes w(y)$ for $a \in A^{*}, y \in Y$.

For any sequence $i_{1}, i_{2}, \ldots, i_{r}$ in $I$ such that $l\left(s_{i_{1}} s_{i_{2}} \ldots s_{i_{r}}\right)=r=\left|R^{+}\right|$, the map $A^{r} \rightarrow G_{A}$ given by

$$
\begin{aligned}
& \left(a_{1}, a_{2}, \ldots, a_{r}\right) \mapsto \\
& x_{i_{1}}\left(a_{1}\right) \dot{s}_{i_{1}} x_{i_{2}}\left(a_{2}\right) \dot{s}_{i_{1}}^{-1} \ldots \dot{s}_{i_{1}} \dot{s}_{i_{2}} \ldots \dot{s}_{i_{r-1}} x_{i_{r}}\left(a_{r}\right) \dot{s}_{i_{r-1}}^{-1} \ldots \dot{s}_{i_{2}}^{-1} \dot{s}_{i_{1}}^{-1}
\end{aligned}
$$

is injective and its image is a subgroup $U_{A}^{+}$of $G_{A}$ independent of the choice of $i_{1}, i_{2}, \ldots, i_{r}$. (See $[\mathrm{L} 18]$.) 
TWELVE BRIDGES FROM A REDUCTIVE GROUP TO ITS LANGLANDS DUAL

Similarly, for any sequence $i_{1}, i_{2}, \ldots, i_{r}$ in $I$ such that $l\left(s_{i_{1}} s_{i_{2}} \ldots s_{i_{r}}\right)=r=$ $\left|R^{+}\right|$, the map $A^{r} \rightarrow G_{A}$ given by

$$
\begin{aligned}
& \left(a_{1}, a_{2}, \ldots, a_{r}\right) \mapsto \\
& y_{i_{1}}\left(a_{1}\right) \dot{s}_{i_{1}} y_{i_{2}}\left(a_{2}\right) \dot{s}_{i_{1}}^{-1} \ldots \dot{s}_{i_{1}} \dot{s}_{i_{2}} \ldots \dot{s}_{i_{r-1}} y_{i_{r}}\left(a_{r}\right) \dot{s}_{i_{r-1}}^{-1} \ldots \dot{s}_{i_{2}}^{-1} \dot{s}_{i_{1}}^{-1}
\end{aligned}
$$

is injective and its image is a subgroup $U_{A}^{-}$of $G_{A}$ independent of the choice of $i_{1}, i_{2}, \ldots, i_{r}$.

The subgroups $U_{A}^{+}, U_{A}^{-}$are normalized by $T_{A}$. We set $B_{A}^{+}=U_{A}^{+} T_{A}=T_{A} U_{A}^{+}$, $B_{A}^{-}=U_{A}^{-} T_{A}=T_{A} U_{A}^{-}$.

If $A$ is a field, we have a partition $G_{A}=\cup_{w \in W} B_{A}^{+} \dot{w} B_{A}^{+}$.

7. Weyl modules. We preserve the notation of $\S 6$. For any $\lambda \in X^{+}$let $\mathcal{T}_{\lambda}=$ $\sum_{i} \underline{f} \theta_{i}^{\left\langle\check{\alpha}_{i}, \lambda\right\rangle+1}$, a left ideal of $\underline{f}$; let $\Lambda_{\lambda}=\underline{f} / \mathcal{T}_{\lambda}$, a finite dimensional Q-vector space. Let $\eta$ be the image of $1 \in f$ in $\Lambda_{\lambda}$. We regard $\Lambda_{\lambda}$ as a $\underline{U}$-module in which $x^{-}$acts as left multiplication by $x(x \in f) ; \theta_{i}^{+} \eta=0$ for $i \in I$; $y \eta=\langle y, \lambda\rangle \eta$ for $y \in Y$. We say that $\Lambda_{\lambda}$ is a Weyl module. $\bar{W}$ e have $\Lambda_{\lambda} \in \mathcal{C}$.

For $\lambda \in X^{+}$let

$$
\mathcal{T}_{\lambda, \mathbf{Z}}=\sum_{i, n ; n \geq\left\langle\check{\alpha}_{i}, \lambda\right\rangle+1} \underline{f} \theta_{i}^{(n)}=\mathcal{T}_{\lambda} \cap \underline{f}_{\mathbf{Z}},
$$

a left ideal of $\underline{f}_{\mathbf{Z}}$. Let $\Lambda_{\lambda, \mathbf{Z}}=\underline{f}_{\mathbf{Z}} / \mathcal{T}_{\lambda, \mathbf{Z}}$. Then $\Lambda_{\lambda, \mathbf{Z}}$ is a lattice in the $\mathbf{Q}$-vector space $\Lambda_{\lambda}$ and a $\underline{U}_{\mathbf{z}}$-submodule of $\Lambda_{\lambda}$. For a commutative ring $A$ with 1 we set $\Lambda_{\lambda, A}=\Lambda_{\lambda, \mathbf{Z}} \otimes A$.

We write $\mathcal{O}^{o p p}, \mathcal{O}_{A}^{o p p}$ for $\mathcal{O}, \mathcal{O}_{A}$ with the opposite comultiplication. Define $\Xi$ : $\Lambda_{\lambda} \rightarrow \mathcal{O} \otimes \Lambda_{\lambda}$ by $e \mapsto \sum_{j} c_{\Lambda_{l}}\left(e \otimes \xi_{j}^{\prime}\right) \otimes e_{j}$ where $\left(e_{j}\right)$ is a Q-basis of $\Lambda_{\lambda}$ and $\left(\xi_{j}\right)$ is the dual basis of $\Lambda_{\lambda}^{\dagger}$. This makes $\Lambda_{\lambda}$ into a $\mathcal{O}^{o p p}$-comodule. Now $\mathcal{O}_{\mathbf{Z}} \otimes_{\mathbf{Z}} \Lambda_{\lambda, \mathbf{Z}}$ is a lattice in $\mathcal{O} \otimes \Lambda_{\lambda}$ and $\Xi$ restricts to $\Xi_{\mathbf{Z}}: \Lambda_{\lambda, \mathbf{Z}} \rightarrow \mathcal{O}_{\mathbf{Z}} \otimes_{\mathbf{Z}} \Lambda_{\lambda, \mathbf{Z}}$. By extension of scalars we obtain an $A$-linear map $\Xi_{A}: \Lambda_{\lambda, A} \rightarrow \mathcal{O}_{A} \otimes_{A} \Lambda_{\lambda, A}$ making $\Lambda_{\lambda, A}$ into a $\mathcal{O}_{A}^{\text {opp }}$-comodule. For any $g: \mathcal{O}_{A} \rightarrow A$ which is in $G_{A}$, we define $\rho_{g}: \Lambda_{\lambda, A} \rightarrow \Lambda_{\lambda, A}$ by $e \mapsto \sum_{h} g\left(f_{h}\right) \otimes e_{h}$ where $\Xi_{A}(e)=\sum_{h} f_{h} \otimes e_{h}, f_{h} \in \mathcal{O}_{A}, e_{h} \in \Lambda_{\lambda, A}$. Note that $g \mapsto \rho_{g}$ is a group action. Thus $\Lambda_{\lambda, A}$ is a $G_{A}$-module.

8. The Langlands dual group. If $A$ is an algebraically closed field then $G_{A}=$ $G_{A}^{\mathcal{R}}$ is a reductive connected algebraic group over $A$ with coordinate $\operatorname{ring} \mathcal{O}_{A}$. Moreover, according to Chevalley, $\mathcal{R} \mapsto G_{A}^{\mathcal{R}}$ is a bijection

$\{$ root systems up to isom. $\} \stackrel{\sim}{\longrightarrow}$

\{reductive connected algebraic groups over $A$ up to isom. .

An element of $G_{A}$ is said to be semisimple if it is conjugate to an element in $T_{A}$. An element of $G_{A}$ is said to be unipotent if it is conjugate to an element in $U_{A}^{+}$. 
(a) In the remainder of these notes (except in $\S 15$ ) we fix a root system $\mathcal{R}=$ $\left(Y, X,\langle\rangle,, \check{\alpha}_{i}, \alpha_{i}(i \in I)\right)$. Define $W, \tilde{W}, \mathcal{H}$ in terms of $\mathcal{R}$ as in $\S 1, \S 3, \S 4$. Define $\tilde{W}^{*}, \mathcal{H}^{*}$ like $\tilde{W}, \tilde{W}^{*}$ but in terms of $\mathcal{R}^{*}$ instead of $\mathcal{R}$.

For a commutative ring $A$ with 1 we write $G_{A}, T_{A}$ instead of $G_{A}^{\mathcal{R}}, T_{A}^{\mathcal{R}}$ and $G_{A}^{*}, T_{A}^{*}$ instead of $G_{A}^{\mathcal{R}^{*}}, T_{A}^{\mathcal{R}^{*}}$. We say that $G_{A}^{*}$ is the Langlands dual group to $G_{A}$. Let $\mathcal{B}_{A}$ be the set of subgroups of $G_{A}$ that are conjugate to $B_{A}^{+}$(or equivalently to $B_{A}^{-}$). Let $\mathcal{B}_{A}^{*}$ be the analogous set defined in terms of $\mathcal{R}^{*}$ instead of $\mathcal{R}$. For $g \in G_{A}^{*}$ we set $\mathcal{B}_{A, g}^{*}=\left\{B \in \mathcal{B}_{A}^{*} ; g \in B\right\}$.

9. Representations of affine Hecke algebras. Assume that $X / \sum_{i} \mathbf{Z} \alpha_{i}$ has no torsion. We fix $\underline{v} \in \mathbf{C}^{*}$. Let $\mathcal{H}_{\underline{v}}=\mathbf{C} \otimes_{\mathcal{A}} \mathcal{H}$ where $\mathbf{C}$ is regarded as an $\mathcal{A}$ -

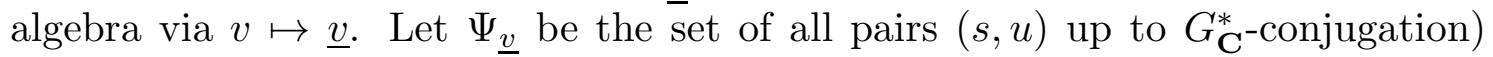
where $s \in G_{\mathbf{C}}^{*}$ is semisimple, $u \in G_{\mathbf{C}}^{*}$ is unipotent and $s u s^{-1}=u \underline{v}^{2}$.

Assume that $\underline{v}$ is not a root of 1 . The Deligne-Langlands conjecture states that there is a canonical finite to one surjective map

$$
\text { \{irred. } \left.\mathcal{H}_{\underline{\underline{v}}} \text {-modules up to isom. }\right\} \rightarrow \Psi_{\underline{v}} \text {. }
$$

A refinement of this conjecture was stated in [L5] namely that the fibre of (a) at $(s, u)$ should be in natural bijection with the set of irreducible representations (up to isomorphism) of the group of connected components of the centralizer of $(s, u)$ in $G_{\mathbf{C}}^{*}$ which appear in the natural representation of this group on the cohomology of $\left\{B \in \mathcal{B}_{\mathbf{C}}^{*} ; s \in B, u \in B\right\}$. This came from a study of examples connected with "subregular" unipotent elements in $G_{\mathbf{C}}^{*}$. In [L7] it was shown that $\mathcal{H}_{\underline{v}}$ acts naturally on the equivariant $K$-theory of $\mathcal{B}_{\mathbf{C}}^{*}$ where the parameter of the $\overline{H e c k e}$ algebra comes from equivariance with respect to a $\mathbf{C}^{*}$-action. In [L7] it was also suggested that one should construct representations of $\mathcal{H}_{\underline{v}}$ using the equivariant $K$-theory of the varieties $\mathcal{B}_{\mathbf{C}, u}^{*}$ for $u \in G_{\mathbf{C}}^{*}$ unipotent. This was established in [KL2] which gave a proof of (a) (in the refined form).

In $[\mathrm{Xi}]$ it is shown (by a reduction to [KL2]) that a statement analogous to (a) (in the refined form) holds also when $\underline{v}$ is allowed to be a root of 1 in the complement of a specific finite set of roots of 1 depending on $\mathcal{R}$.

10. $p$-adic groups. Let $K$ be a finite extension of the field of $p$-adic numbers $(p$ a prime number). Let $\mathfrak{a}$ be the integral closure in $K$ of the ring of $p$-adic integers and let $\mathfrak{m}$ be the unique maximal ideal of $\mathfrak{a}$ so that $\mathfrak{a} / \mathfrak{m}$ is a finite field with $q$ elements. Let $\bar{K}$ be an algebraic closure of $K$. Let $\overline{\mathfrak{a}}$ be the integral closure of $\mathfrak{a}$ in $\bar{K}$ and let $\overline{\mathfrak{m}}$ be the unique maximal ideal of $\overline{\mathfrak{a}}$ so that $\overline{\mathfrak{a}} / \overline{\mathfrak{m}}$ is an algebraic closure of $\mathfrak{a} / \mathfrak{m}$. Let $W_{K}$ be the Weil group of $K$ that is the inverse image under the natural homomorphism $\pi: \operatorname{Gal}(\bar{K} / K) \rightarrow \operatorname{Gal}(\overline{\mathfrak{a}} / \overline{\mathfrak{m}}, \mathfrak{a} / \mathfrak{m})$ of the subgroup $\mathbf{Z}$ of $\operatorname{Gal}(\overline{\mathfrak{a}} / \overline{\mathfrak{m}}, \mathfrak{a} / \mathfrak{m})$ consisting of the integer powers of the automorphism $x \mapsto x^{q}$. Let $\mathcal{I}$ be the kernel of $\pi$. We have an exact sequence $1 \rightarrow \mathcal{I} \rightarrow W_{K} \stackrel{\omega}{\rightarrow} \mathbf{Z} \rightarrow 1$ where $\omega$ is the restriction of $\pi$. A homomorphism $\rho: W_{K} \rightarrow G_{\mathbf{C}}^{*}$ is said to be admissible if $\rho(\mathcal{I})$ is finite and $\rho(\phi)$ is semisimple in $G_{\mathbf{C}}^{*}$ for some/any $\phi \in \omega^{-1}(1)$. Let 
$\Phi^{K}\left(G_{\mathbf{C}}^{*}\right)$ be the set of all pairs $(\rho, u)$ (up to $G_{\mathbf{C}^{-}}^{*}$ conjugacy) where $\rho: W_{K} \rightarrow G_{\mathbf{C}}^{*}$ is an admissible homomorphism and $u \in G_{\mathbf{C}}^{*}$ is a unipotent element such that $\rho(w) u \rho(w)^{-1}=u^{q^{\omega(w)}}$ for any $w \in W_{K}$. We regard $G_{K}$ as a topological group with the $p$-adic topology. An irreducible representation $G_{K} \rightarrow G L(E)$ (where $E$ is a $\mathbf{C}$-vector space) is said to be admissible if the stabilizer of any vector of $E$ is open in $G_{K}$ and if for any open subgroup $H$ of $G_{K}$ the space of $H$-invariant vectors in $E$ has finite dimension. According to the local Langlands conjecture there is a canonical finite to one surjective map

(a) $\quad$ irred. admissible representations of $G_{K}$ up to isom. $\} \rightarrow \Phi^{K}\left(G_{\mathbf{C}}^{*}\right)$.

This is known to be true in the case where $G_{K}=G L_{n}(K)$, see [HT]. In the general case but assuming that $\mathcal{R}$ is adjoint, a class of irreducible admissible representations (called "unipotent") has been decribed in [L15] where a canonical finite to one surjective map

$$
\text { \{unipotent representations of } \left.G_{K} \text { up to isom. }\right\} \rightarrow \Phi_{1}^{K}\left(G_{\mathbf{C}}^{*}\right)
$$

was constructed; here $\Phi_{1}^{K}\left(G_{\mathbf{C}}^{*}\right)=\left\{(\rho, u) \in \Phi\left(G_{\mathbf{C}}^{*}\right) ; \rho(\mathcal{I})=\{1\}\right\}$. Note that $\Phi_{1}^{K}\left(G_{\mathbf{C}}^{*}\right)$ may be identified with the set $\Psi_{\sqrt{q}}$ in $\S 9$ and that (b) constitutes a verification of (a) in a special case. Note that some of the unipotent representations can be understood by the method described in $\S 9$; to understand the remaining ones one needs the theory of character sheaves and a geometric construction of certain affine Hecke algebras with unequal parameters in terms of equivariant homology.

For any $(\rho, u) \in \Phi^{K}\left(G_{\mathbf{C}}^{*}\right)$ we denote by $\Xi_{\rho, u}$ the set of irreducible representations (up to isomorphism) of the group of connected components of the simultaneous centralizer of $(\rho, u)$ in $G_{\mathbf{C}}^{*}$ on which the action of the centre of $G_{\mathbf{C}}^{*}$ is trivial.

According to [L15], for any $(\rho, u) \in \Phi_{1}^{K}\left(G_{\mathbf{C}}^{*}\right)$ the fibre of the map (b) at $(\rho, u)$ is in bijection with $\Xi_{\rho, u}$. This suggests that more generally for any $(\rho, u) \in \Phi^{K}\left(G_{\mathbf{C}}^{*}\right)$, the fibre of the (conjectural) map (a) at $(\rho, u)$ is in bijection with $\Xi_{\rho, u}$.

Note that in general neither side of (a) is well undestood. But recent results of J.-L.Kim [Ki] give a classification of "supercuspidal representations" of $G_{K}$ (assuming that $p$ is sufficiently large) which gives some hope that the left hand side of (a) can be understood for such $p$.

11. Real groups. The Weil group of $\mathbf{R}$ is by definition $W_{\mathbf{R}}=\mathbf{C}^{*} \times \operatorname{Gal}(\mathbf{C} / \mathbf{R})$ with the group structure $\left(z_{1}, \tau_{1}\right)\left(z_{2}, \tau_{2}\right)=\left((-1)^{\epsilon\left(\tau_{1}\right) \epsilon\left(\tau_{2}\right)} z_{1} \tau_{1}\left(z_{2}\right), \tau_{1} \tau_{2}\right)$ where $\epsilon(\tau)=0$ if $\tau=1$ and $\epsilon(\tau)=1$ if $\tau \neq 1$. We can identify $W_{\mathbf{R}}$ with the subgroup of the group of nonzero quaternions $a+b i+c j+d k$ generated by $\left\{a+b i ;(a, b) \in \mathbf{R}^{2}-\{0\}\right\}$ and by $j$. We regard $W_{\mathbf{R}}$ as a Lie group with two connected components. Let $\Phi^{\mathbf{R}}\left(G_{\mathbf{C}}^{*}\right)$ be the set of all continuous homomorphism $W_{\mathbf{R}} \rightarrow G_{\mathbf{C}}^{*}$ whose image consists of semisimple elements, up to conjugation by $G_{\mathbf{C}}^{*}$. Let $\mathcal{K}$ be a maximal compact subgroup subgroup of the Lie group $G_{\mathbf{R}}$. An "irreducible admissible" representation of $G_{\mathbf{R}}$ is by definition a $\mathbf{C}$-vector space $E$ 
with an action of $\mathcal{K}$ and one of the Lie $\left(G_{\mathbf{R}}\right)$ such that any vector in $E$ is contained in a finite dimensional $\mathcal{K}$-stable subspace of $E$; the two actions induce the same action on Lie $(\mathcal{K})$; the action of Lie $\left(G_{\mathbf{R}}\right)$ is compatible with the $\mathcal{K}$-action on Lie $\left(G_{\mathbf{R}}\right)$ and the $\mathcal{K}$-action on $E$. Moreover, this should be irreducible in the obvious sense.

According to Langlands [La2] there is a canonical finite to one surjective map

(a) $\quad$ irred. admissible representations of $G_{\mathbf{R}}$ up to isom. $\} \rightarrow \Phi^{\mathbf{R}}\left(G_{\mathbf{C}}^{*}\right)$.

The fibres of (a) have been described by Knapp and Zuckerman.

We now give some examples of elements of $\Phi^{\mathbf{R}}\left(G_{\mathbf{C}}^{*}\right)$ in the case where $G_{\mathbf{C}}^{*}=$ $G L(V)$ with $V$ a finite dimensional $\mathbf{C}$-vector space. Assume that we are given a direct sum decomposition $V=\oplus_{p, q} V^{p, q}$ with $(p, q) \in \mathbf{C} \times \mathbf{C}, p-q \in \mathbf{Z}$; assume also that we are given a $\mathbf{C}$-linear isomorphism $\phi: V \rightarrow V$ such that $\phi^{2}=1$ and $\phi\left(V^{p, q}\right)=V^{q, p}$ for all $p, q$. We define an action of $W_{\mathbf{R}}$ on $V$ by specifying the action of $(z, 1)$ with $z \in \mathbf{C}^{*}$ and the action of $(1, \tau)$ with $\tau \in \operatorname{Gal}(\mathbf{C} / \mathbf{R})-\{1\}$. If $(p, q)$ runs only in $\mathbf{Z} \times \mathbf{Z}$ we have $(z, 1) \cdot x=z^{p} \bar{z}^{q} x$ for $z \in \mathbf{C}^{*}, x \in V^{p, q}$. The same formula holds in the general case: we interpret $z^{p} \bar{z}^{q}$ as $(z \bar{z})^{(p+q) / 2}\left(\frac{z}{\sqrt{z \bar{z}}}\right)^{p-q}$. (The strictly positive real number $z \bar{z}$ can be raised to any complex power.) We have $(1, \tau) \cdot x=\sqrt{(-1)^{p-q}} \phi(x)$ for $x \in V^{p, q}$. This is an object of $\Phi^{\mathbf{R}}\left(G_{\mathbf{C}}^{*}\right)$.

12. Global fields. Let $k$ be a field which is a finite algebraic extension of the field of rational functions in one variable over the finite field $\mathbf{F}_{p}$. Let $A$ be the ring of adeles of $k$ and let $k \rightarrow A$ be the canonical imbedding. Let $\bar{k}$ be an algebraic closure of $k$. Let $l$ be a prime number $\neq p$.

The global Langlands conjecture [La1] predicts a connection between the set consisting of irreducible "cuspidal" representations of $G_{A}$ with nonzero vectors fixed by $G_{k}$ on the one hand and a certain set of homomorphisms of $\operatorname{Gal}(\bar{k} / k)$ into $G_{\mathbf{Q}_{l}}^{*}$ which are irreducible in a suitable sense, on the other hand.

This conjecture has been proved in the case where $G_{k}=G L_{n}(k)$. (See [Dr] for $n=2$ and [Lf] for any $n$.)

There is an analogous conjecture in which $k$ is replaced by a finite extension of $\mathbf{Q}$ and also a geometric analogue of the conjecture in which the curve over a finite field represented by $k$ is replaced by a smooth projective curve over $\mathbf{C}$. (See $[\mathrm{KW}]$.

13. Unipotent classes and cells in affine Weyl groups. Define $\phi: \mathcal{H} \rightarrow$ $\mathcal{A} \otimes J$ in terms of $\mathcal{R}$ as in $\S 4$. Let $K$ be an algebraic closure of the field $\mathbf{C}(v)$ of rational functions with coefficients in $\mathbf{C}$ in an indeterminate $v$. Let $c$ be a two-sided cell of $\tilde{W}$. Let $J_{c}$ be the corresponding direct summand of the ring $J$. We can find some simple module $E$ of the $\mathbf{C}$-algebra $\mathbf{C} \otimes J_{c}$. It is necessarily of finite dimension over $\mathbf{C}$. We can regard $K \otimes_{\mathbf{C}} E$ as a $K \otimes_{\mathbf{Q}} J$-module in which the summands $K \otimes_{\mathbf{Q}} J_{c^{\prime}}$ act as zero for $c^{\prime} \neq c$. For $y, y^{\prime}$ in $Y^{+}$we have $T_{a^{y}} T_{a^{y^{\prime}}}=T_{a^{y^{\prime}}} T_{a^{y}}=T_{a^{y+y^{\prime}}}$ (see $\S 3$ ). Hence the operators $\phi\left(T_{a^{y}}\right): K \otimes_{\mathbf{C}} E \rightarrow K \otimes_{\mathbf{C}} E$ (with $y \in Y^{+}$) commute. 
We can find $e \in K \otimes_{\mathbf{C}} E-\{0\}$ which is a simultaneous eigenvector for these operators. Thus we have $\phi\left(T_{a^{y}}\right) e=b(y) e$ for all $y \in Y^{+}$where $b(y) \in K^{*}$ satisfy $b(y) b\left(y^{\prime}\right)=b\left(y+y^{\prime}\right)$ for any $y, y^{\prime}$ in $Y^{+}$. There is a unique element $t \in K^{*} \otimes X$ such that, if $t=\sum_{s} k_{s} \otimes x_{s}$ with $k_{s} \in K^{*}, x_{s} \in X$ then $b(y)=\prod_{s} k_{s}^{\left\langle y, x_{\rangle}\right\rangle}$for any $y \in Y^{+}$. One can show [L9] that $t$ is a very special element of $K^{*} \otimes T$ : we can write uniquely $t=t^{\prime} t^{\prime \prime}$ where $t^{\prime \prime} \in C^{*} \otimes X$ and $t^{\prime} \in\left\{v^{n} ; n \in \mathbf{Z}\right\} \otimes X \subset T_{K}^{*} \subset G_{K}^{*}$ is equal to $\phi\left(\begin{array}{cc}v & 0 \\ 0 & v^{-1}\end{array}\right)$ for some homomorphism of algebraic groups $\phi: S L_{2}(K) \rightarrow G_{K}^{*}$. Let $C$ be the conjugacy class in $G_{\mathbf{C}}^{*}$ such that $\phi\left(\begin{array}{ll}1 & 1 \\ 0 & 1\end{array}\right)$ is conjugate in $G_{K}^{*}$ to some element of $C$. One can show [L9] that $C$ is well defined by $c$ (it is independent of the choice of $E, e, \phi)$ and that $c \mapsto C$ is a

(a) bijection $\{$ two-sided cells of $\tilde{W}\} \stackrel{\sim}{\longrightarrow}\left\{\right.$ unipotent conjugacy classes in $\left.G_{\mathbf{C}}^{*}\right\}$.

14. Special unipotent classes. We preserve the setup in $\S 13$. The intersection of $W$ with a two-sided cell of $\tilde{W}$ is said to be a two-sided cell of $W$ if it is nonempty. Note that the two-sided cells of $W$ form a partition of $W$. A unipotent conjugacy class in $G_{\mathbf{C}}^{*}$ is said to be special if it corresponds under the bijection $\S 13($ a) to a two-sided cell of $\tilde{W}$ which has a nonempty intersection with $W$.

The special unipotent classes of $G_{\mathbf{C}}^{*}$ were introduced in a different (but equivalent) way in [L1] as the unipotent classes such that the corresponding irreducible representation of $W$ (under the Springer correspondence) is in the class $\mathcal{S}_{W}$ defined in [L1]. This definition makes sense when $\mathbf{C}^{*}$ is replaced by any algebraically closed field $A$. For $\rho \in \mathcal{S}_{W}$ we denote by $C_{\rho, A}$ the corresponding special unipotent element of $G_{A}$ and by $C_{\rho, A}^{*}$ the corresponding special unipotent element of $G_{A}^{*}$. (The sets $\mathcal{S}_{W}$ for $\mathcal{R}, \mathcal{R}^{*}$ coincide.) Let $\hat{C}_{\rho, A}=\bar{C}_{\rho, A}-\cup_{C^{\prime}} \bar{C}^{\prime}$ where $\bar{C}_{\rho, A}$ is the closure of $C_{\rho, A}$ and $C^{\prime}$ runs over the special unipotent classes contained in $\bar{C}_{\rho, A}-C_{\rho, A}$. It is known that the subsets $\hat{C}_{\rho, A}$ form a partition of the unipotent variety of $G_{A}$ into locally closed subvarieties which are rational homology manifolds. We define similarly the subvarieties $\hat{C}_{\rho, A}^{*}$ of the unipotent variety of $G_{A}^{*}$. We have the following result (see [L7],[L16]):

For any $\rho \in \mathcal{S}_{W}$ there exists a polynomial $P_{\rho}$ with integer coefficients such that for any $q$ (a power of a prime number) we have

$$
\left|\hat{C}_{\rho, \overline{\mathbf{F}}_{q}} \cap G_{\mathbf{F}_{q}}\right|=\left|\hat{C}_{\rho, \overline{\mathbf{F}}_{q}}^{*} \cap G_{\mathbf{F}_{q}}^{*}\right|=P_{\rho}(q) .
$$

15. Preparatory results. Let $Y, X$ be two free abelian groups of finite rank and let $\langle\rangle:, Y \times X \rightarrow \mathbf{Z}$ be a perfect pairing. Let $\mathfrak{A}: Y \rightarrow Y$ be a homomorphism such that $\operatorname{det}(\mathfrak{A}) \neq 0$ that is such that $|Y / \mathfrak{A} Y|<\infty$. We then have $|Y / \mathfrak{A} Y|= \pm \operatorname{det}(\mathfrak{A})$. Define a homomorphism $\mathfrak{A}^{\prime}: X \rightarrow X$ by $\left\langle y, \mathfrak{A}^{\prime}(x)\right\rangle=\langle\mathfrak{A}(y), x\rangle$ for all $y \in Y, x \in$ $X$. Then $\operatorname{det}\left(\mathfrak{A}^{\prime}\right)=\operatorname{det}(\mathfrak{A})$ hence $\left|X / \mathfrak{A}^{\prime}(X)\right|<\infty$. Now $\mathfrak{A}$ (resp. $\left.\mathfrak{A}^{\prime}\right)$ induces endomorphisms of $\mathbf{Q} \otimes Y$ and of $\mathbf{Q} / \mathbf{Z} \otimes Y$ (resp. $\mathbf{Q} \otimes X$ and $\mathbf{Q} / \mathbf{Z} \otimes X$ ) denoted 
again by $\mathfrak{A}\left(\right.$ resp. $\left.\mathfrak{A}^{\prime}\right)$. Also, $\langle$,$\rangle induces a \mathbf{Q}$-linear pairing $(\mathbf{Q} \otimes Y) \times(\mathbf{Q} \otimes X) \rightarrow \mathbf{Q}$ denoted again by $\langle$,$\rangle . We define a pairing ():, Y / \mathfrak{A}(Y) \times X / \mathfrak{A}^{\prime}(X) \rightarrow \mathbf{Q} / Z Z$ by

$$
(y, x)=\left\langle\mathfrak{A}^{-1}(y), x\right\rangle \bmod \mathbf{Z}=\left\langle y, \mathfrak{A}^{\prime-1}(x)\right\rangle \quad \bmod \mathbf{Z}
$$

where $y \in Y, \mathfrak{A}^{-1}(y) \in \mathbf{Q} \otimes Y, x \in X, \mathfrak{A}^{\prime-1}(x) \in \mathbf{Q} \otimes X$. Now $x \mapsto[y \mapsto(y, x)]$ is an isomorphism

(a) $X / \mathfrak{A}^{\prime}(X) \stackrel{\sim}{\rightarrow} \operatorname{Hom}(Y / \mathfrak{A}(Y), \mathbf{Q} / \mathbf{Z})$.

We define an isomorphism $Y / \mathfrak{A}(Y) \stackrel{\sim}{\longrightarrow}(\mathbf{Q} / \mathbf{Z} \otimes Y)^{\mathfrak{A}+1}$ (fixed point set of $\mathfrak{A}+1$ ) by

$$
y \mapsto \text { image of } \mathfrak{A}^{-1}(y) \text { under } \mathbf{Q} \otimes Y \rightarrow \mathbf{Q} / \mathbf{Z} \otimes Y \text {. }
$$

Similarly we have an isomorphism $X / \mathfrak{A}^{\prime}(X) \stackrel{\sim}{\longrightarrow}(\mathbf{Q} / \mathbf{Z} \otimes X)^{\mathfrak{A}^{\prime}+1}$. Via the last two isomorphisms, (a) becomes an isomorphism

$$
(\mathbf{Q} / \mathbf{Z} \otimes X)^{\mathfrak{A}^{\prime}+1} \stackrel{\sim}{\longrightarrow} \operatorname{Hom}\left((\mathbf{Q} / \mathbf{Z} \otimes Y)^{\mathfrak{A}+1}, \mathbf{Q} / \mathbf{Z}\right)
$$

This is induced by $\xi \mapsto\left[\eta \mapsto\langle\mathfrak{A}(\eta), \xi\rangle \bmod \mathbf{Z}\right.$ where $\xi \in \mathbf{Q} \otimes X, \mathfrak{A}^{\prime}(\xi) \in X$, $\eta \in \mathbf{Q} \otimes Y, \mathfrak{A}(\eta) \in Y$. Let $p$ be a prime number and let $(\mathbf{Q} / \mathbf{Z})^{\prime}$ be the subgroup of $\mathbf{Q} / \mathbf{Z}$ consisting of elements of order not divisible by $p$. Assume now that $p$ does not divide $\operatorname{det}(\mathfrak{A})$. Then $(\mathbf{Q} / \mathbf{Z} \otimes Y)^{\mathfrak{A}+1}=\left((\mathbf{Q} / \mathbf{Z})^{\prime} \otimes Y\right)^{\mathfrak{A}+1}$ and we get an isomorphism

$$
(\mathbf{Q} / \mathbf{Z} \otimes X)^{\mathfrak{A}^{\prime}+1} \stackrel{\sim}{\longrightarrow} \operatorname{Hom}\left(\left((\mathbf{Q} / \mathbf{Z})^{\prime} \otimes Y\right)^{\mathfrak{A}+1},(\mathbf{Q} / \mathbf{Z})^{\prime}\right)
$$

Now let $k$ be an algebraic closure of the finite field $\mathbf{F}_{p}$. Let $\left(k^{*} \otimes Y\right)^{\mathfrak{A}+1}$ be the fixed point set of the endomorphism $z \otimes y \mapsto z \otimes(\mathfrak{A}+1) y$ of $k^{*} \otimes Y$. We define a canonical isomorphism

$$
\operatorname{Hom}\left(\left((\mathbf{Q} / \mathbf{Z})^{\prime} \otimes Y\right)^{\mathfrak{A}+1},(\mathbf{Q} / \mathbf{Z})^{\prime}\right) \stackrel{\sim}{\longrightarrow} \operatorname{Hom}\left(\left(k^{*} \otimes Y\right)^{\mathfrak{A}+1}, k^{*}\right)
$$

as follows. We choose an isomorphism $\zeta:(\mathbf{Q} / \mathbf{Z})^{\prime} \stackrel{\sim}{\rightarrow} k^{*}$. Then $\zeta \otimes 1:(\mathbf{Q} / \mathbf{Z})^{\prime} \otimes$ $Y) \stackrel{\sim}{\longrightarrow} k^{*} \otimes Y$ restricts to an isomorphism $\left.\zeta_{1}:(\mathbf{Q} / \mathbf{Z})^{\prime} \otimes Y\right)^{\mathfrak{A}+1} \stackrel{\sim}{\longrightarrow}\left(k^{*} \otimes Y\right)^{\mathfrak{A}+1}$ and (c) carries a homomorphism $\phi:\left((\mathbf{Q} / \mathbf{Z})^{\prime} \otimes Y\right)^{\mathfrak{A}+1} \rightarrow(\mathbf{Q} / \mathbf{Z})^{\prime}$ to $\zeta \phi \zeta_{1}^{-1}$. We must show that the map (c) is independent of the choice of $\zeta$. Let $\kappa:(\mathbf{Q} / \mathbf{Z})^{\prime} \stackrel{\sim}{\longrightarrow}$ $(\mathbf{Q} / \mathbf{Z})^{\prime}$ be an isomorphism. Then $\left.\kappa \otimes 1:(\mathbf{Q} / \mathbf{Z})^{\prime} \otimes Y\right) \stackrel{\sim}{\longrightarrow}(\mathbf{Q} / \mathbf{Z})^{\prime} \otimes Y$ restricts to an isomorphism $\left.\kappa_{1}:(\mathbf{Q} / \mathbf{Z})^{\prime} \otimes Y\right)^{\mathfrak{A}+1} \stackrel{\sim}{\longrightarrow}\left((\mathbf{Q} / \mathbf{Z})^{\prime} \otimes Y\right)^{\mathfrak{A}+1}$ and it is enough to show that for any homomorphism $\phi:\left((\mathbf{Q} / \mathbf{Z})^{\prime} \otimes Y\right)^{\mathfrak{A}+1} \rightarrow(\mathbf{Q} / \mathbf{Z})^{\prime}$ we have $\kappa \phi \kappa_{1}^{-1}=\phi$. Since $(\mathbf{Q} / \mathbf{Z})^{\prime}$ is an injective $\mathbf{Z}$-module, there exists a homomorphism $\tilde{\phi}:(\mathbf{Q} / \mathbf{Z})^{\prime} \otimes Y \rightarrow(\mathbf{Q} / \mathbf{Z})^{\prime}$ whose restriction to $\left((\mathbf{Q} / \mathbf{Z})^{\prime} \otimes Y\right)^{\mathfrak{A}+1}$ is $\phi$. It is enough to show that $\kappa \tilde{\phi}(\kappa \otimes 1)^{-1}=\tilde{\phi}$. By choosing a basis of $Y$ we see that it is enough to show that for any homomorphism $\psi:(\mathbf{Q} / \mathbf{Z})^{\prime} \rightarrow(\mathbf{Q} / \mathbf{Z})^{\prime}$ we have $\kappa \psi \kappa^{-1}=\psi$. This follows from the fact that the ring of endomorphisms of the group $(\mathbf{Q} / \mathbf{Z})^{\prime}$ is commutative (it is a product of rings of $l$-adic integers for various primes $l \neq p$ ). 
Let $\mu$ be the group of roots of 1 in $\mathbf{C}$. We note that the isomorphism $\zeta^{\prime}: \mathbf{Q} / \mathbf{Z} \rightarrow$ $\mu$ given by $r \mapsto \exp (2 \pi i r)$ induces an isomorphism $\zeta^{\prime} \otimes 1: \mathbf{Q} / \mathbf{Z} \otimes X \rightarrow \mu \otimes X$ and this restricts to an isomorphism $\zeta_{1}^{\prime}:(\mathbf{Q} / \mathbf{Z} \otimes X)^{\mathfrak{A}^{\prime}+1} \stackrel{\sim}{\longrightarrow}(\mu \otimes X)^{\mathfrak{A}^{\prime}+1}$ where $(\mu \otimes X)^{\mathfrak{A}^{\prime}+1}$ is the fixed point set of the endomorphism $z \otimes x \mapsto z \otimes\left(\mathfrak{A}^{\prime}+1\right) x$ of $\mu \otimes X$. Via $\zeta_{1}^{\prime}$ and (c), the isomorphism (b) becomes a canonical isomorphism

$$
(\mu \otimes X)^{\mathfrak{A}^{\prime}+1} \stackrel{\sim}{\longrightarrow} \operatorname{Hom}\left(\left(k^{*} \otimes Y\right)^{\mathfrak{A}+1}, k^{*}\right) .
$$

16. Groups over $\mathbf{F}_{q}$. We return to the setup in $\S 8(\mathrm{a})$. Let $k$ be an algebraic closure of the finite field $\mathbf{F}_{p}$. Let $K$ be an algebraically closed field of characteristic 0 with a fixed imbedding of groups $\iota: k^{*} \rightarrow K^{*}$.

We have $T_{k}=k^{*} \otimes Y, T_{\mathbf{C}}^{*}=\mathbf{C}^{*} \otimes X$. Let $\mathbf{F}_{q}$ be the subfield of $k$ such that $\left|\mathbf{F}_{q}\right|=q$. The ring homomorphism $k \rightarrow k, c \mapsto c^{q}$ induces (as in §6) a group homomorphism $F: G_{k} \rightarrow G_{k}$ (Frobenius map) whose fixed point set is the finite group $G_{\mathbf{F}_{q}}$. Following [DL] we consider for any $w \in W$ the set $\dot{X}_{w}=$ $\left\{g \in G_{k} ; g^{-1} F(g) \in \dot{w} U_{k}^{+}\right\}$, an algebraic variety over $k$. Let $T_{k}^{w}=\left\{t \in T_{k} ; t^{q}=\right.$ $\left.w^{-1}(t)\right\}$, a finite subgroup of $T_{k}$. The finite group $G_{\mathbf{F}_{q}} \times T_{k}^{w}$ acts on $\dot{X}_{w}$ by $\left(g_{1}, t\right): g \mapsto g_{1} g t^{-1}$. Let $\chi_{w}: G_{\mathbf{F}_{q}} \times T_{k}^{w} \rightarrow \mathbf{Z}$ be the class function which to any $\left(g_{1}, t\right) \in G_{\mathbf{F}_{q}} \times T_{k}^{w}$ associates the alternating sum of traces of $\left(g_{1}, t\right)^{*}$ on the $l$-adic cohomology with compact support of $\dot{X}_{w}$. (Here $l$ is any prime number $\neq p$ but the resulting class function is known to be independent of $l$; see [DL].) For any irreducible $G_{\mathbf{F}_{q}}$-module $\rho$ over $K$ let $\mathcal{E}_{\rho}$ be the set of all pairs $(w, \theta)$ where $w \in W$, $\theta \in \operatorname{Hom}\left(T_{k}^{w}, K^{*}\right)$ and $\sum_{\left(g_{1}, t\right) \in G_{\mathbf{F}_{q}} \times T_{k}^{w}} \theta(t) \operatorname{tr}\left(g_{1}, \rho\right) \chi_{w}\left(g_{1}, t\right) \neq 0$. According to [DL] we have $\mathcal{E}_{\rho} \neq \emptyset$ for any $\rho$.

To any $(w, \theta) \in \mathcal{E}_{\rho}$ we associate an element $\hat{\theta} \in T_{\mathbf{C}}^{* w^{-1}}:=\left\{t \in T_{\mathbf{C}}^{*} ; t^{q}=w(t)\right\}$ as follows. Define $\mathfrak{A}: Y \rightarrow Y$ by $y \mapsto q w(y)-y$ and $\mathfrak{A}^{\prime}: X \rightarrow X$ by $x \mapsto$ $q w^{-1}(x)-x$. Then $T_{k}^{w}=\left(k^{*} \otimes Y\right)^{\mathfrak{A}+1}$ is a finite group of order prime to $p$. Hence $\theta: T_{k}^{w} \rightarrow K^{*}$ has values in the group of roots of 1 of order prime to $p$ in $K^{*}$ which can be identified with $k^{*}$ via $\iota$. Thus $\theta$ can be viewed as an element of $\operatorname{Hom}\left(\left(k^{*} \otimes Y\right)^{\mathfrak{A}+1}, k^{*}\right)$ so that it corresponds under $\S 15(\mathrm{~d})$ to an element $\hat{\theta}$ of $(\mu \otimes X)^{\mathfrak{A}^{\prime}+1}$. This last group is a subgroup of $\left(\mathbf{C}^{*} \otimes X\right)^{\mathfrak{A}^{\prime}+1}$ (the fixed point set of the endomorphism $z \otimes x \mapsto z \otimes\left(\mathfrak{A}^{\prime}+1\right) x$ of $\left.\mathbf{C}^{*} \otimes X\right)$ which is the same as $T_{\mathbf{C}}^{* w^{-1}}$. From the results in [DL] we see that the $W$-orbit of $\hat{\theta}$ in $T_{\mathbf{C}}^{*}$ depends only on $\rho$ and not on the choice of $(w, \theta)$ in $\mathcal{E}_{\rho}$. We thus have a well defined map

\{irred. $G_{\mathbf{F}_{q}}$-modules over $K$ up to isom. $\} \rightarrow$

$$
\left\{\text { semisimple conjugacy classes in } G_{\mathbf{C}}^{*} \text { stable under } g \mapsto g^{q}\right. \text { \}; }
$$

it is given by $\rho \mapsto G_{\mathbf{C}^{-}}^{*}$-conjugacy class of $\hat{\theta}$ (as above). This map appears in [DL] in a somewhat different form. In $[\mathrm{DL}] G_{\mathbf{C}}^{*}$ is replaced by $G_{k}^{*}$. But the method of [DL] is less canonical: it is based on two choices (see [DL, $(5.0 .1),(5,0.2)]$ ) while the present method is based on only one choice, that of $\iota$; the choice of $\iota$ can be also eliminated as we will see below). 
An element $g \in G_{\mathbf{C}}^{*}$ is said to be special if the unipotent part $g_{u}$ of $g$ is a special unipotent element (see $\S 14$ ) of the connected centralizer of the semisimple part $g_{s}$ of $g$ (a reductive connected group). A conjugacy class in $G_{\mathbf{C}}^{*}$ is said to be special if some/any element of it is special. The map (a) can be refined to a canonical map

\{irred. $G_{\mathbf{F}_{q}}$-modules over $K$ up to isom. $\} \rightarrow$

(See [L6].) Note that (a) is the composition of (b) with the map which to the $G_{\mathbf{C}^{-}}^{*}$-conjugacy class of a special element $g$ associates the $G_{\mathbf{C}^{-}}^{*}$-conjugacy class of $g_{s}$. The map (b) is surjective and its fibres are described explicitly in [L6], [L10].

Note that the maps (a),(b) depend on the choice of the imbedding $\iota: k^{*} \rightarrow K^{*}$. However if we take $K$ to be an algebraic closure of the quotient field of the ring of Witt vectors of $k$ then there is a canonical choice of $\iota$ and the maps (a),(b) become completely canonical.

17. Character sheaves. Define $B_{\mathbf{C}}^{+}, U_{\mathbf{C}}^{+}, \dot{w}$ in terms of $\mathbf{C}, \mathcal{R}$ as in $\S 6$. Let $\mathcal{E}$ be a C-local system of rank 1 on $T_{\mathbf{C}}$ with finite monodromy. The monodromy of $\mathcal{E}$ is a homomorphism $f: Y \rightarrow \mathbf{C}^{*}$ with finite image which can be viewed as an element of finite order $\chi_{\mathcal{E}} \in \mathbf{C}^{*} \otimes X$ given by $\chi_{\mathcal{E}}=\sum_{j} f\left(y_{j}\right) \otimes x_{j}$ where $\left(y_{j}\right)$ is a basis of $Y$ and $\left(x_{j}\right)$ is the dual basis of $X$. Moreover $\mathcal{E} \mapsto \chi_{\mathcal{E}}$ is a bijection

$\left\{\mathbf{C}-\right.$ local systems of rank 1 on $T_{\mathbf{C}}$ with finite monodromy up to isom. $\} \stackrel{\sim}{\longrightarrow}$ (a)

\{elements of finite order of $\left.T_{\mathbf{C}}^{*}\right\}$.

Let $c: G_{\mathbf{C}} \rightarrow G_{\mathbf{C}} / U_{\mathbf{C}}^{+}$be the obvious map. An irreducible intersection cohomology complex $K$ on $G_{\mathbf{C}}$ is said to be a character sheaf on $G_{\mathbf{C}}$ if it is $G_{\mathbf{C}^{-}}$equivariant and if for any $w \in W$ and any $j \in \mathbf{Z}$ the $j$-th cohomology sheaf of $c_{!} K$ restricted to $B_{\mathbf{C}}^{+} \dot{w} B_{\mathbf{C}}^{+} / U_{\mathbf{C}}^{+}$is a local system $\mathcal{L}_{K, w, j}$ with finite monodromy. We can find $w, j$ as above and a local system $\mathcal{E}$ of rank 1 on $T_{\mathbf{C}}$ with finite monodromy such that $\mathcal{E}$ is a direct summand of the inverse image of $\mathcal{L}_{K, w, j}$ under the map $T_{\mathbf{C}} \rightarrow B_{\mathbf{C}}^{+} \dot{w} B_{\mathbf{C}}^{+} / U_{\mathbf{C}}^{+}$, $t \mapsto \dot{w} t U_{\mathbf{C}}^{+}$. One can show that the corresponding element $\chi_{\mathcal{E}} \in T_{\mathbf{C}}^{*}$ is well defined (up to the action of $W$ ) that is, it does not depend on the choice of $w, j, \mathcal{E}$. Thus we have a well defined map

$\left\{\right.$ character sheaves on $G_{\mathbf{C}}$ up to isom. $\rightarrow \rightarrow$

$\left\{\right.$ conjugacy classes of elements of finite order in $\left.G_{\mathbf{C}}^{*}\right\}$;

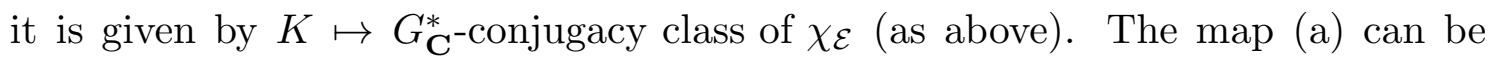
refined to a canonical map

$\left\{\right.$ character sheaves on $G_{\mathbf{C}}$ up to isom. $\} \rightarrow$

\{special conjugacy classes in $G_{\mathbf{C}}^{*}$ of elements $g$ with $g_{s}$ of finite order . 
(See [L8].) Note that (a) is the composition of (b) with the map which to the $G_{\mathbf{C}^{-}}^{*}$-conjugacy class of a special element $g$ associates the $G_{\mathbf{C}^{-}}^{*}$ conjugacy class of $g_{s}$. The map (b) is surjective and its fibres are described explicitly in [L8].

18. Vogan duality. To $G_{\mathbf{C}}$ we associate a finite collection of polynomials: those recording the restrictions of the cohomology sheaves of the simple perverse sheaves on $\mathcal{B}_{\mathbf{C}}$, equivariant under the conjugation action of the centralizers of the various "inner" involutions of $G_{\mathbf{C}}$, to the various orbits of these centralizers. (These polynomials were studied in [LV].) We consider also the analogous collection of polynomials associated to $G_{\mathbf{C}}^{*}$. Vogan duality $[\mathrm{Vo}]$ states that these two collections of polynomials are related to each other by a simple algebraic rule: essentially the inversion of a matrix. This is a generalization of the inversion formula in [KL1].

19. Multiplicities in standard modules. Let $K$ be either as in $\S 10$ or $K=\mathbf{R}$. The Grothendieck group whose basis consists of admissible irreducible representations of $G_{K}$ has another natural basis consisting of "standard representations". The representations in the second basis are easier to describe and understand. Hence the (upper triangular) matrix expressing the second basis in terms of the first basis ("multiplicity matrix") is of interest. In every known case the entries of this matrix can be expressed in terms of intersection cohomology coming from the geometry of $G_{\mathbf{C}}^{*}$. For the case where $K=\mathbf{R}$ we refer the reader to $[\mathrm{ABV}]$; in this case some of the polynomials in $\S 18$ (attached to $G_{\mathbf{C}}^{*}$ ) evaluated at 1 appear as entries of this matrix. In the remainder of this subsection we assume that $K$ is as in $\S 10$. We use the notation in $\S 10$. We fix an element $w^{0} \in W_{K}$ such that $\omega\left(w^{0}\right)=1$. Let $\tilde{\Phi}^{K}$ be the set of all triples $(\rho, u, E$ ) (up to $G_{\mathbf{C}^{-}}^{*}$-conjugacy) where $\rho: W_{K} \rightarrow G_{\mathbf{C}}^{*}$ is an admissible homomorphism, $u$ is a unipotent element of $G_{\mathbf{C}}^{*}$ such that $\rho(w) u \rho(w)^{-1}=u^{q^{\omega(w)}}$ for all $w \in W_{K}$ and $E$ is an irreducible representation of the group of connected components of $G_{\rho, u}^{*}:=\left\{g \in G_{\mathbf{C}}^{*} ; \rho(w) g \rho(w)^{-1}=g\right.$ for all $\left.w \in W_{K}, g u=u g\right\}$ on which the image of the centre of $G_{\mathbf{C}}^{*}$ acts trivially.

By $\S 10$ it is expected that $\tilde{\Phi}^{K}$ is an index set for both the irreducible admissible representations and the standard representations of $G_{K}$ (up to isomorphism). We shall describe a matrix indexed by $\tilde{\Phi}^{K}$ which is defined in terms of geometry of $G_{\mathbf{C}}^{*}$ and is expected to coincide with the multiplicity matrix above. (This was conjectured in a special case connected with $G L_{n}$ in [Ze] and independently by the author.)

Let $\Psi$ be the set of homomorphisms $\psi: \mathcal{I} \rightarrow G_{\mathbf{C}}^{*}$ such that $\psi(\mathcal{I})$ is finite and such that $\Gamma_{\psi}:=\left\{g \in G_{\mathbf{C}}^{*} ; g \psi(w) g^{-1}=\psi\left(w^{0} w w^{0-1}\right)\right.$ for all $\left.w \in \mathcal{I}\right\}$ is non-empty. Let $\bar{\Psi}$ be the set of $G_{\mathrm{C}^{-}}^{*}$-orbits (by conjugacy) on $\Psi$.

Define $\kappa: \tilde{\Phi}^{K} \rightarrow \bar{\Psi}$ by $\left.(\rho, u, E) \mapsto \rho\right|_{\mathcal{I}}$. The entries $m_{(\rho, u, E),\left(\rho^{\prime}, u^{\prime}, E^{\prime}\right)}$ of our matrix can be described as follows. If $(\rho, u, E),\left(\rho^{\prime}, u^{\prime}, E^{\prime}\right)$ are not in the same fibre of $\kappa$ then $m_{(\rho, u, E),\left(\rho^{\prime}, u^{\prime}, E^{\prime}\right)}=0$.

We now fix $\psi \in \Psi$. Let $G_{\psi}^{*}=\left\{g \in G_{\mathbf{C}}^{*} ; g \psi(w) g^{-1}=\psi(w)\right.$ for all $\left.w \in \mathcal{I}\right\}$. This is the centralizer of a finite subgroup of $G_{\mathbf{C}}^{*}$ hence is a (possibly disconnected) 
reductive subgroup of $G_{\mathbf{C}}^{*}$. Let $G_{\psi}^{\prime}{ }^{*}$ be the normalizer of $\psi(\mathcal{I})$ in $G_{\mathbf{C}}^{*}$. Note that $G_{\psi}^{*}$ is a normal subgroup of finite index of $G_{\psi}^{\prime}{ }^{*}$ and that $\Gamma_{\psi}$ is a single $G_{\psi^{-}}^{*}$ coset in $G_{\psi}^{\prime}{ }^{*}$. Let $\mathfrak{g}_{\psi}$ be the Lie algebra of $G_{\psi}^{*}$. The fibre of $\kappa$ at $\psi$ can be identified with the set of all triples $(s, N, E)$ (up to $G_{\psi}^{*}$-conjugacy) where $s \in G_{\psi}^{\prime}{ }^{*}$ is a semisimple element such that $s \in \Gamma_{\psi}, N$ is an element of $X_{s}:=\left\{N_{1} \in\right.$ $\left.\mathfrak{g}_{\psi} ; \operatorname{Ad}(s) N_{1}=q N\right\}$ (necessarily nilpotent) and $E$ is an irreducible representation of the group of connected components of $G_{\psi, s, N}^{*}:=\left\{g \in G_{\psi}^{*} ; g s=s g, \operatorname{Ad}(g) N=\right.$ $N\}$ on which the image of the centre of $G_{\mathbf{C}}^{*}$ acts trivially. (The identification is given by $(\rho, u, E) \mapsto\left(\rho\left(w^{0}\right), \log (u), E\right)$ where $\left.\rho\right|_{\mathcal{I}}=\psi$.) We now consider two elements $(s, N, E),\left(s^{\prime}, N^{\prime}, E^{\prime}\right)$ in $\kappa^{-1}(\psi)$. If $s, s^{\prime}$ are not in the same $G_{\psi}^{*}$-orbit then $m_{(s, N, E),\left(s^{\prime}, N^{\prime}, E^{\prime}\right)}=0$. Now assume that $(s, N, E),\left(s^{\prime}, N^{\prime}, E^{\prime}\right)$ are such that $s, s^{\prime}$ are in the same $G_{\psi}^{*}$-orbit. We can assume that $s=s^{\prime}$. Let $G_{\psi, s}^{*}=\left\{g \in G_{\psi}^{*} ; g s=\right.$ $s g\}$. This is an algebraic group which acts on $X_{s}$ by conjugation with finitely many orbits. Let $C$ be the $G_{\psi, s}^{*}$-orbit of $N$ and let $C^{\prime}$ be the $G_{\psi, s}^{*}$-orbit of $N^{\prime}$. Note that $E$ (resp. $E^{\prime}$ ) determines a local system $\underline{E}$ (resp. $\underline{E}^{\prime}$ ) on $C$ (resp. $C^{\prime}$ ) which is $G_{\psi, s}^{*}$-equivariant and is irreducible as a $G_{\psi, s}^{*}$-equivariant local system. If $C$ is not contained in the closure of $C^{\prime}$ then $m_{(s, N, E),\left(s^{\prime}, N^{\prime}, E^{\prime}\right)}=0$. Now assume that $C$ is contained in the closure of $C^{\prime}$. Let $\underline{E}^{\prime \sharp}$ be the intersection cohomology complex on the closure of $C^{\prime}$ determined by $\underline{E}^{\prime}$. For every integer $j$ we consider the $j$-th cohomology sheaf of $\underline{E}^{\sharp \sharp}$ restricted to $C$; this is a $G_{\psi, s}^{*}$-equivariant local system on $C$ in which $\underline{E}$ appears say $n_{j}$ times. We set $m_{(s, N, E),\left(s^{\prime}, N^{\prime}, E^{\prime}\right)}=\sum_{j}(-1)^{j} n_{j}$.

We see that the intersection cohomology complexes on $X_{s}$ considered above are essentially of the type considered in [L17]. We also see that the objects in $\kappa^{-1}(\psi)$ behave like the parameters for the unipotent representations for a collection of not necessarily split and not necessarily connected $p$-adic groups smaller than $G_{K}$.

20. Multiplicities in tensor products. Assume that $\mathcal{R}$ is simply connected. For $\lambda, \lambda^{\prime}, \lambda^{\prime \prime}$ in $X^{+}$let $m_{\lambda, \lambda^{\prime}, \lambda^{\prime \prime}}$ be the multiplicity of $\Lambda_{\lambda^{\prime \prime}}$ in the tensor product $\Lambda_{\lambda} \otimes \Lambda_{\lambda^{\prime}}$ (an object of $\mathcal{C}$, see $\left.\S 5\right)$. On the other hand let $l^{*}: \tilde{W}^{*} \rightarrow \mathbf{N}, T_{w}, c_{w}, p_{w, z}$ be defined like $l: \tilde{W} \rightarrow \mathbf{N}, T_{w}, c_{w}, p_{w, z}$ in $\S 3, \S 4$ but with respect to $\mathcal{R}^{*}$ instead of $\mathcal{R}$. We have $\tilde{W}^{*}=\left\{w a^{x} ; w \in W, x \in X\right\}$.

For any $\lambda \in X^{+}$there is a unique element $M_{\lambda}$ in the double coset $W a^{\lambda} W$ on which $l^{*}: W a^{\lambda} W \rightarrow \mathbf{N}$ achieves its maximum value. For any $\lambda, \lambda^{\prime}$ in $X^{+}$we have in $\mathcal{H}^{*}$ :

$$
\left(P^{-1} c_{M_{\lambda}}\right)\left(P^{-1} c_{M_{\lambda^{\prime}}}\right)=\sum_{\lambda^{\prime \prime} \in X^{+}} \tilde{m}_{\lambda, \lambda^{\prime}, \lambda^{\prime \prime}}\left(P^{-1} c_{M_{\lambda^{\prime \prime}}}\right)
$$

where $P \in \mathcal{A}$ is given by $c_{M_{0}} c_{M_{0}}=P c_{M_{0}}$ and $\tilde{m}_{\lambda, \lambda^{\prime}, \lambda^{\prime \prime}} \in \mathcal{A}$. In [L4] it is shown that

$$
m_{\lambda, \lambda^{\prime}, \lambda^{\prime \prime}}=\tilde{m}_{\lambda, \lambda^{\prime}, \lambda^{\prime \prime}}
$$

in particular,

$$
\tilde{m}_{\lambda, \lambda^{\prime}, \lambda^{\prime \prime}} \text { is a constant. }
$$


(In the special case where $G_{\mathbf{C}}$ is a general linear group, this was proved earlier in [L3] using the theory of Hall-Littlewood functions.) In [L4] it is also shown that for $\lambda, \lambda^{\prime \prime}$ in $X^{+}$,

(c) $\operatorname{dim} \Lambda_{\lambda}^{\lambda^{\prime}}$ (that is the multiplicity of the weight $\lambda^{\prime}$ in the $\underline{U}$-module $\Lambda_{\lambda}$ ) is equal to $p_{M_{l^{\prime}}, M_{\lambda}}(1)$.

Note that at the time when [L4] was written it was known that the product of elements of the form $P^{-1} c_{M_{\lambda}}$ corresponds to the convolution of $G_{\mathbf{C}[[\epsilon]]}^{*}$-equivariant simple perverse sheaves on the "affine Grassmannian" $G_{\mathbf{C}((\epsilon))}^{*} / G_{\mathbf{C}[[\epsilon]]}^{*}$ so that (b) is equivalent to the statement that such a convolution is a direct sum of simple perverse sheaves of the same type (without shift). Thus it was clear that the category whose objects are finite direct sums of $G_{\mathbf{C}[[\epsilon]]}^{*}$-equivariant simple perverse sheaves on the "affine Grassmannian" has a natural monoidal structure given by convolution; moreover (b) showed that this monoidal category was very similar to that of representations of $G_{\mathbf{C}}$ (identical at the level of Grothendieck groups). But it was not clear how to construct the commutativity isomorphism for the convolution product. This was accomplished around 1989 by V.Ginzburg [Gi] and later in a more elegant form by V.Drinfeld. As a result, $G_{\mathbf{C}}$ can be reconstructed from the tensor category of $G_{\mathbf{C}[[\epsilon]]}^{*}$-equivariant perverse sheaves on $G_{\mathbf{C}((\epsilon))}^{*} / G_{\mathbf{C}[[\epsilon]]}^{*}$, see $[\mathrm{Gi}]$.

21. Canonical bases. Define $f$ as in $\S 5$ in terms of $\mathcal{R}$. Let $A=\mathbf{R}[[\epsilon]]$ where $\epsilon$ is an indeterminate. Define $U_{A}^{*+}$ in terms of $\mathcal{R}^{*}$ in the same way as $U_{A}^{+}$was defined in $\S 6$ in terms of $\mathcal{R}$. By $[\mathrm{L} 14, \S 10]$ there is a canonical bijection between the canonical basis of $\underline{f}$ (defined as in [L11], [L13]) and a certain collection of subsets of $U_{A}^{*+}$ which form a partition of the totally positive part of $U_{A}^{*+}$. The bijection is not defined directly; instead it is shown that both sets are parametrized by the same combinatorial objects.

22. Modular representations. Let $k$ be an algebraic closure of the finite field $\mathbf{F}_{p}$. Assume that $\mathcal{R}$ is simply connected. For $\lambda \in X^{+}$the $G_{k}$-module $\Lambda_{\lambda, k}$ (see $\S 7)$ is not necessarily irreducible but has a unique irreducible quotient $\Lambda_{\lambda, k}^{\sharp}$. For $\lambda, \lambda^{\prime}$ in $X^{+}$let $m_{l, \lambda^{\prime}}$ be the number of times that $\Lambda_{\lambda^{\prime}, k}^{\sharp}$ appears in a composition series of the $G_{k}$-module $\Lambda_{\lambda, k}$. Note that the knowledge of the multiplicities $m_{\lambda, \lambda^{\prime}}$ implies the knowledge of the character of the $G_{k}$-modules $\Lambda_{\lambda, k}^{\sharp}$ since the character of $\Lambda_{\lambda, k}$ is known by Weyl's character formula. Conjecturally (see [L2]) if $p$ is sufficiently large with respect to $\mathcal{R}$, the multiplicities $m_{\lambda, \lambda^{\prime}}$ can be expressed in terms of polynomials $p_{w, z}$ (as in $\S 4$ ) where $w, z$ are elements in $\tilde{W}^{*}$ which have maximal length in their left $W$-coset; they can be also expressed in terms of certain intersection cohomology spaces associated with the geometry of $G_{\mathbf{C}((\epsilon))}^{*}$, where $\epsilon$ is an indeterminate. A proof of the conjecture (without an explicit bound for $p$ ) is provided by combining [AJS], [KT], [KL3] or alternatively by combining [AJS], [ABG]. 


\section{REFERENCES}

[ABV] J.Adams, D.Barbasch and D.Vogan, The Langlands classification of irreducible characters of real reductive groups, Progress in Math (1992), Birkhauser.

[AJS] H.Andersen, W.Soergel and J.Jantzen, Representations of quantum groups at a $p$-th root of unity and of semisimple groups in characteristic $p$ : independence of $p$, Astérisque $\mathbf{2 2 0}$ (1994).

[ABG] S.Arkhipov, R.Bezrukavnikov and V.Ginzburg, Quantum groups, the loop grassmannian and the Springer resolution, Jour.Amer.Math.Soc. 17 (2004), 595-678.

[C] C.Chevalley, Certains schémas de groupes semi-simples, Sém. Bourbaki 13ème année, 1960-1961.

[DL] P.Deligne and G.Lusztig, Representations of reductive groups over finite fields, Ann. Math. 103 (1976), 103-161.

[Dr] V.Drinfeld, Two dimensional l-adic representations of the fundamental group of a curve over a finite field and automorphic forms on GL(2), Amer.Jour.Math. 105 (1983), 85114.

[Gi] V.Ginzburg, Perverse sheaves on a loop group and Langlands duality, math.AG/9511007.

[HT] M.Harris and R. Taylor, On the geometry and cohomology of some simple Shimura varieties, Ann.Math.Studies, Princeton Univ.Press, 2001.

[IM] N.Iwahori and H.Matsumoto, On some Bruhat decomposition and the structure of the Hecke ring of p-adic Chevalley groups, Publ.Math.IHES 25 (1965), 5-48.

[KW] A.Kapustin and E.Witten, Electric-magnetic duality and the geometric Langlands program, arXiv:hep-th/0604151.

[KT] M.Kashiwara and T.Tanisaki, The Kazhdan-Lusztig conjecture for affine Lie algebras with negative level, Duke Math.J. 77 (1995), 21-62.

[KL1] D.Kazhdan and G.Lusztig, Representations of Coxeter groups and Hecke algebras, Invent.Math. 53 (1979), 165-184.

[KL2] D.Kazhdan and G.Lusztig, Proof of the Deligne-Langlands conjecture for Hecke algebras, Invent.Math. 87 (1987), 153-215.

[KL3] D.Kazhdan and G.Lusztig, Tensor structures arising from affine Lie algebras, IV, Jour. Amer. Math. Soc. 7 (1994), 383-453.

[Ki] J.-L.Kim, Supercuspidal representations: an exhaustion theorem, Jour.Amer.Math.Soc. 20 (2007), 273-320.

[Ko] B.Kostant, Groups over Z, Algebraic groups and discontinuous subgroups, Proc. Symp. Pure Math., vol. 9 Amer. Math. Soc, 1966.

[Lf] L.Lafforgue, Chtoukas de Drinfeld et correspondance de Langlands, Invent.Math. 147 (2002), 1-242.

[La1] R.Langlands, Problems in the theory of automorphic forms, Lectures in Modern Analysis and Applications, Lecture Notes in Math, vol. 170, Springer Verlag, 1970.

[La2] R.Langlands, On the classification of irreducible representations of real algebraic groups, Institute for Advanced Study (1973).

[L1] G.Lusztig, A class of irreducible representations of a Weyl group, Proc. Kon. Nederl. Akad. (A) 82 (1979), 323-335.

[L2] G.Lusztig, Some problems in the representation theory of finite Chevalley groups, Proc. Symp. Pure Math. Amer. Math. Soc. 37 (1980), 313-317.

[L3] G.Lusztig, Green polynomials and singularities of unipotent classes, Adv. Math. 42 (1981), 169-178.

[L4] G.Lusztig, Singularities, character formulas and a q-analog of weight multiplicities, Astérisque 101-102 (1983), 208-229.

[L5] G.Lusztig, Some examples of square integrable representations of semisimple p-adic groups, Trans.Amer.Math.Soc. 227 (1983), 623-653. 
TWELVE BRIDGES FROM A REDUCTIVE GROUP TO ITS LANGLANDS DUAL 21

[L6] G.Lusztig, Characters of reductive groups over a finite field, Ann.Math.Studies, vol. 107, Princeton Univ.Press, 1984.

[L7] G.Lusztig, Equivariant $K$-theory and representations of Hecke algebras, Proc. Amer. Math. Soc. 94 (1985), 337-342.

[L8] G.Lusztig, Character sheaves, V, Adv.Math. 61 (1986), 103-155.

[L9] G.Lusztig, Cells in affine Weyl groups, II, J.Alg. 109 (1987), 536-548; IV, J. Fac. Sci. Tokyo U.(IA) 36 (1989), 297-328.

[L10] G.Lusztig, On representations of reductive groups with disconnected center, Astérisque 168 (1988), 157-166.

[L11] G.Lusztig, Canonical bases arising from quantized enveloping algebras, Jour. Amer. Math. Soc. 3 (1990), 447-498.

[L12] G.Lusztig, Affine quivers and canonical bases, Publ.Math.IHES 76 (1992), 111-163.

[L13] G.Lusztig, Introduction to quantum groups, Progress in Math., vol. 110, Birkhauser, 1993.

[L14] G.Lusztig, Total positivity in reductive groups, Lie theory and geometry, Progr.in Math., vol. 123, Birkhäuser Boston, 1994.

[L15] G.Lusztig, Classification of unipotent representations of simple p-adic groups, Int. Math. Res. Notices (1995), 517-589; II, Represent. Th. 6 (2002), 243-289.

[L7] G.Lusztig, Notes on unipotent classes, Asian J.Math. 1 (1997), 194-207.

[L16] G.Lusztig, Unipotent elements in small characteristic, Transform. Groups 10 (2005), 449-487; II,arXiv:RT/0612320.

[L17] G.Lusztig, Graded Lie algebras and intersection cohomology, arXiv:RT/0604535.

[L18] G.Lusztig, Study of a $\mathbf{Z}$-form of the coordinate ring of a reductive group.

[LV] G.Lusztig and D.Vogan, Singularities of closures of $K$-orbits on a flag manifold, Invent.Math. 71 (1983), 365-379.

[MK] J.McKay, Graphs, singularities and finite groups 37 (1980), 183-186.

[SL] P.Slodowy, Simple singularities and simple algebraic groups, Lecture Notes in Math., vol. 815, Springer Verlag, 1980.

[Vo] D.Vogan, Irreducible characters of semisimple Lie groups, IV: character multiplicity duality, Duke Math.J. 4 (1982), 943-1073.

[Xi] N.Xi, Representations of affine Hecke algebras and based rings of affine Weyl groups, Jour.Amer.Math.Soc. 20 (2007), 211-217.

[Ze] A.Zelevinsky, A p-adic analogue of the Kazhdan-Lusztig conjecture, Funkt.Anal.Pril. 15 (1981), 9-21.

Department of Mathematics, M.I.T., Cambridge, MA 02139 